\title{
Semidefinite bounds for the stability number of a graph via sums of squares of polynomials
}

\author{
Nebojša Gvozdenović • Monique Laurent
}

Received: 27 June 2005 / Accepted: 4 April 2006 /

Published online: 15 December 2006

(C) Springer-Verlag 2006

\begin{abstract}
Lovász and Schrijver (SIAM J. Optim. 1:166-190, 1991) have constructed semidefinite relaxations for the stable set polytope of a graph $G=$ $(V, E)$ by a sequence of lift-and-project operations; their procedure finds the stable set polytope in at most $\alpha(G)$ steps, where $\alpha(G)$ is the stability number of $G$. Two other hierarchies of semidefinite bounds for the stability number have been proposed by Lasserre (SIAM J. Optim. 11:796-817, 2001; Lecture Notes in Computer Science, Springer, Berlin Heidelberg New York, pp 293-303, 2001) and by de Klerk and Pasechnik (SIAM J. Optim. 12:875-892), which are based on relaxing nonnegativity of a polynomial by requiring the existence of a sum of squares decomposition. The hierarchy of Lasserre is known to converge in $\alpha(G)$ steps as it refines the hierarchy of Lovász and Schrijver, and de Klerk and Pasechnik conjecture that their hierarchy also finds the stability number after $\alpha(G)$ steps. We prove this conjecture for graphs with stability number at most 8 and we show that the hierarchy of Lasserre refines the hierarchy of de Klerk and Pasechnik.
\end{abstract}

Keywords Stability number of a graph $\cdot$ Semidefinite programming $\cdot$ Sum of squares of polynomials

Mathematics Subject Classification (2000) $\quad$ 05C69 • 90C22 · 90C27

\footnotetext{
N. Gvozdenović · M. Laurent (ه)

Centrum voor Wiskunde en Informatica, Kruislaan 413, 1098 SJ Amsterdam, The Netherlands

e-mail: monique@cwi.nl

N. Gvozdenović

e-mail: nebojsa@cwi.nl
} 


\section{Introduction}

Semidefinite programming plays an essential role for constructing good relaxations for hard combinatorial optimization problems, in particular, for the maximum stable set problem which will be considered in the present paper (see, e.g., [11] for a detailed account). Lovász [12] introduced the theta number $\vartheta(G)$ as an upper bound for the stability number $\alpha(G)$ of a graph $G=(V, E)$. The theta number can be formulated via the semidefinite program

$$
\vartheta(G):=\max \operatorname{Tr}(J X) \text { s.t. } \operatorname{Tr}(X)=1, \quad X_{i j}=0(i j \in E), X \succeq 0,
$$

and thus computed efficiently (to any arbitrary precision) using, e.g., interior point methods (cf. $[2,25]$ ). It is also known that $\vartheta(G)$ coincides with $\alpha(G)$ when $G$ is a perfect graph (see [6]). Lovász and Schrijver [13] construct a hierarchy of semidefinite relaxations for the stable set polytope of $G$ by a sequence of lift-and-project operations; their procedure is finite and it finds the stable set polytope in at most $\alpha(G)$ steps.

Two other hierarchies of semidefinite bounds for the stability number have been proposed by Lasserre [7,8] and by de Klerk and Pasechnik [5]. They use the following notions about of sum of squares of polynomials: given a polynomial $f \in \mathbb{R}\left[x_{1}, \ldots, x_{n}\right]$, one says that $f$ is a sum of squares of polynomials if it can be written as $f=g_{1}^{2}+g_{2}^{2}+\cdots+g_{m}^{2}$, where $g_{1}, \ldots, g_{m}$ are polynomials in $\mathbb{R}\left[x_{1}, \ldots, x_{n}\right]$. Obviously, $f$ is nonnegative on $\mathbb{R}^{n}$ if it can be written as sum of squares of polynomials. Hierarchies of Lasserre and of de Klerk and Pasechnik are based on the following paradigm: while testing nonnegativity of a polynomial is a hard problem, one can test efficiently whether a polynomial can be written as a sum of squares of polynomials via semidefinite programming. As was already proved by Hilbert in 1888 not every nonnegative multivariate polynomial can be written as a sum of squares (see Reznick [19] for a nice survey on this topic). However, some representation theorems have been proved ensuring the existence of certain sums of squares decompositions under some assumption, like positivity of the polynomial on a compact basic closed semi-algebraic set (see, e.g., [24] for an exposition of such results). An early such result is due to Pólya [18] who showed that, if $p(x)$ is a homogeneous polynomial which is positive on $\mathbb{R}_{+}^{n} \backslash\{0\}$, then $\left(\sum_{i=1}^{n} x_{i}\right)^{r} p(x)$ has only nonnegative coefficients [and thus $\left(\sum_{i=1}^{n} x_{i}^{2}\right)^{r} p\left(x_{1}^{2}, \ldots, x_{n}^{2}\right)$ is a sum of squares] for some sufficiently large integer $r$.

The starting point for Lasserre's construction is that the stability number $\alpha(G)$ of a graph $G=(V, E)$ can be expressed as the smallest scalar $t$ for which the polynomial $t-\sum_{i \in V} x_{i}$ is nonnegative on the set $\left\{x \in \mathbb{R}^{V} \mid x_{i} x_{j}=0(i j \in E)\right.$, $\left.x_{i}^{2}=x_{i}(i \in V)\right\}$. Requiring the weaker condition that the polynomial $t-\sum_{i \in V} x_{i}$ can be written as a sum of squares modulo the ideal generated by $x_{i} x_{j}(i j \in E)$ and $x_{i}^{2}-x_{i}(i \in V)$ with given degree bounds, yields a hierarchy of semidefinite upper bounds for $\alpha(G)$. The dual approach (in terms of moment matrices) yields the hierarchy of Lasserre $[7,8]$ of semidefinite relaxations for the stable 
set polytope. This hierarchy refines the hierarchy of Lovász and Schrijver (see [9]) and thus it also finds the stable set polytope in $\alpha(G)$ steps.

By a result of Motzkin and Straus [15], one may alternatively express $\alpha(G)$ as the smallest scalar $t$ for which the matrix $M:=t\left(I+A_{G}\right)-J$ (with entries $t-1$ on the diagonal and at positions corresponding to edges and -1 elsewhere) is copositive, meaning that the polynomial $p_{M}(x):=\sum_{i, j \in V} x_{i}^{2} x_{j}^{2} M_{i j}$ is nonnegative on $\mathbb{R}^{n}$. Following Parrilo [16], de Klerk and Pasechnik [5] propose to relax the nonnegativity condition on $p_{M}(x)$ and to require instead that $\left(\sum_{i \in V} x_{i}^{2}\right)^{r} p_{M}(x)$ be a sum of squares for some integer $r \geq 0$. In this way they define a hierarchy of bounds $\vartheta^{(r)}(G)$ (for $r \geq 0$ ). The convergence of these bounds to $\alpha(G)$ is guaranteed by the above mentioned result of Pólya. The first bound in the hierarchy coincides with the strengthening $\vartheta^{\prime}(G)$ of the theta number introduced by McEliece et al. [14] and Schrijver [21] (see (7) below). It is however not clear how the next bounds relate to the bounds provided by the construction of Lasserre. It is conjectured in [5] that the stability number is found after $\alpha(G)$ steps. In this paper, we study this conjecture and develop a proof technique which enables us to show that the conjecture holds for graphs with stability number at most 8. Moreover, we show that the hierarchy of bounds of Lasserre (enhanced by adding some nonnegativity constraint) refines the hierarchy of bounds of de Klerk and Pasechnik, answering another open question of [5].

The paper is organized as follows. In Sect. 2, we first recall some definitions and results related to the hierarchies of bounds of Lasserre and of de Klerk and Pasechnik. Next we introduce a dual formulation for the latter bounds, as well as a weighted analogue and new semidefinite relaxations of the stable set polytope. We complete the section with our main results. The proofs are delayed till Sect. 3, where we prove the conjecture for graphs with stability number at most 8 , till Sect. 4, where we prove a partial result for the weighted analogue of the conjecture, and till Sect. 5, where we prove the relation between the hierarchies of Lasserre and of de Klerk and Pasechnik. Section 6 contains some variations and new interpretations of the bounds $\vartheta^{(r)}(G)$. Finally, some variations of the main conjecture about the convergence to the stability number in $\alpha(G)$ steps are given.

Some notation Throughout, $G=(V, E)$ denotes a graph with node set $V=\{1, \ldots, n\}$. For a node $i \in V, N(i)$ denotes the set of nodes adjacent to $i$ and we set $i^{\perp}:=i \cup N(i)$. Similarly for $S \subseteq V, N(S)$ denotes the set of nodes adjacent to some node in $S$ and we set $S^{\perp}:=S \cup N(S)$. For two nodes $u, v \in V$, write $u \simeq v$ if $u=v$ or $u v \in E$, and $u \not z v$ otherwise. Let $\alpha(G)$ denote the stability number of $G$, i.e., the largest cardinality of a stable set in $G$. The matrix $A_{G}$ denotes the adjacency matrix of $G$, i.e., $A_{G}$ is the $0 / 1$ matrix indexed by $V$ whose $(i, j)$-th entry is 1 when $i j \in E$. All matrices are assumed to be symmetric and $I, J, e, e_{i}$ $(i=1, \ldots, n)$ denote, respectively, the identity matrix, the all-ones matrix, the all-ones vector, and the standard unit vectors of suitable sizes. A matrix $M$ is copositive if $x^{\mathrm{T}} M x \geq 0$ for all $x \in \mathbb{R}_{+}^{n}$ and $\mathcal{C}_{n}$ denotes the copositive cone, consisting of the $n \times n$ copositive matrices. For a symmetric matrix $M$, we write $M \geq 0$ if all entries are nonnegative, and $M \succeq 0$ if $M$ is positive semidefinite. The 
trace of $M$ is denoted by $\operatorname{Tr}(M)$, while $\operatorname{diag}(M)$ denotes the vector containing the diagonal entries of $M$. Given a vector $v \in \mathbb{R}^{n}$, we let $\operatorname{Diag}(v)$ denote the diagonal matrix whose diagonal entries are the components of $v$. Next, $\frac{1}{v}$ denotes the vector with entries $\frac{1}{v_{i}}(i=1, \ldots, n)$. For a sequence $\beta \in \mathbb{Z}_{+}^{n}$, we set $|\beta|:=\sum_{i=1}^{n} \beta_{i}$, $\beta !:=\beta_{1} ! \cdots \beta_{n} !, S(\beta):=\left\{i \mid \beta_{i} \neq 0\right\}$, and $S_{\text {odd }}(\beta):=\left\{i \mid \beta_{i}\right.$ is odd $\}$. One says that $\beta$ is even when $S_{\text {odd }}(\beta)=\emptyset$. We also set $I(n, r):=\left\{\beta \in \mathbb{Z}_{+}^{n}|| \beta \mid=r\right\}$ and $\mathcal{P}_{r}(V):=\{S \subseteq V|| S \mid \leq r\}$. For $x \in \mathbb{R}^{n}$ and $\beta \in I(n, r)$ we write $x^{\beta}:=\Pi_{i=1}^{n} x_{i}^{\beta_{i}}$. Following this a polynomial of the form $p(x)=\sum_{\beta \in I(n, r)} p_{\beta} x^{\beta} \in \mathbb{R}[x]$ is said to be homogeneous of degree $r$, and we let $p=\left(p_{\beta}\right) \in \mathbb{R}^{I(n, r)}$ denote the vector containing its coefficients. For a cone of symmetric matrices $\mathcal{K} \subseteq \mathbb{R}^{n \times n}, \mathcal{K}^{*}$ denote the dual cone defined by $\mathcal{K}^{*}=\left\{M \in \mathbb{R}^{n \times n} \mid \operatorname{Tr}(M N) \geq 0, \forall N \in \mathcal{K}\right\}$. It is well known that the cone of positive semidefinite matrices is self-dual (i.e., coincides with its dual cone), while the dual cone of the copositive cone $\mathcal{C}_{n}$ is the cone of completely positive matrices. We call a matrix $M$ doubly nonnegative if $M \succeq 0$ and $M \geq 0$.

\section{Semidefinite bounds for the stability number}

\subsection{The semidefinite bounds of Lasserre}

Given an integer $r \geq 1$ and a vector $x=\left(x_{I}\right)_{I \in \mathcal{P}_{2 r}(V)}$, consider the matrix

$$
M_{r}(x):=\left(x_{I \cup J}\right)_{I, J \in \mathcal{P}_{r}(V)}
$$

known as the moment matrix of $x$ of order $r$. By setting

one obtains a hierarchy of semidefinite bounds for the stability number, known as Lasserre's hierarchy $[8,9]$. Indeed, if $S$ is a stable set, the vector $x \in \mathbb{R}^{\mathcal{P}_{2 r}(V)}$ with $x_{I}=1$ if $I \subseteq S$ and $x_{I}=0$ otherwise, is feasible for (2) with objective value $|S|$, showing $\alpha(G) \leq \operatorname{las}^{(1)}(G)$. We note that $\operatorname{las}^{(1)}(G)=\vartheta^{\prime}(G)$ (see (7) below for the definition of $\vartheta^{\prime}(G)$ ). Note that the conditions $x_{I} \geq 0$ for $|I| \leq r$ are implied by $M_{r}(x) \succeq 0$. For any fixed $r$, the parameter las ${ }^{(r)}(G)$ can be computed in polynomial time (to an arbitrary precision) since the semidefinite program (2) involves matrices of size $O\left(n^{r}\right)$ with $O\left(n^{2 r}\right)$ variables.

Equality $\alpha(G)=$ las $^{(r)}(G)$ holds for $r \geq \alpha(G)$. This result remains valid if we remove the nonnegativity constraint: $x_{I} \geq 0(|I|=r+1)$ in (2) ([9]). However, with this nonnegativity condition, we will be able to compare the hierarchies of Lasserre and of de Klerk and Pasechnik (see Theorem 4 below $^{1}$ ).

\footnotetext{
1 There exist graphs with $\vartheta(G)>\vartheta^{\prime}(G)$ (see, e.g., [22, vol. B, p. 1173]). Therefore, without condition $x_{I} \geq 0(|I|=r+1)$ for $r=1$ we would have $\operatorname{las}^{(1)}(G)=\vartheta(G) \geq \vartheta^{\prime}(G)=\vartheta^{(0)}(G)$, implying that the statement of the theorem is wrong.
} 
2.2 The semidefinite bounds of de Klerk and Pasechnik

The starting point in [5] is the following formulation for $\alpha(G)$ found by Motzkin and Straus [15]

$$
\frac{1}{\alpha(G)}=\min x^{\mathrm{T}}\left(I+A_{G}\right) x \quad \text { subject to } x \geq 0, \sum_{i=1}^{n} x_{i}=1 \text {. }
$$

In other words,

$$
\alpha(G)=\min t \quad \text { subject to } t\left(I+A_{G}\right)-J \in \mathcal{C}_{n} .
$$

Therefore, upper bounds for $\alpha(G)$ can be obtained by replacing the copositive cone $\mathcal{C}_{n}$ in program (3) by a smaller subcone of it. Following [5,16], given an integer $r \geq 0, \mathcal{K}_{n}^{(r)}$ denotes the cone of $n \times n$ matrices $M$ for which the polynomial

$$
p_{M}^{(r)}(x):=\left(\sum_{i=1}^{n} x_{i}^{2}\right)^{r}\left(\sum_{i, j=1}^{n} M_{i j} x_{i}^{2} x_{j}^{2}\right)
$$

can be written as a sum of squares of polynomials. Parrilo [16] shows that

$$
\mathcal{K}_{n}^{(0)}=\{P+N \mid P \succeq 0, N \geq 0\}
$$

A characterization of $\mathcal{K}_{n}^{(1)}$ can be found in [1,16] (see Lemma 14 and the comment thereafter). Obviously, $\mathcal{K}_{n}^{(r)} \subseteq \mathcal{K}_{n}^{(r+1)} \subseteq \cdots \subseteq \mathcal{C}_{n}$. The result of Pólya mentioned in Sect. 1 shows that the interior of the cone $\mathcal{C}_{n}$ is contained in $\bigcup_{r \geq 0} \mathcal{K}_{n}^{(r)}$. Setting

$$
\vartheta^{(r)}(G):=\min \quad t \text { subject to } t\left(I+A_{G}\right)-J \in \mathcal{K}_{n}^{(r)}
$$

one obtains a hierarchy of upper bounds for $\alpha(G)$. The first bound $\vartheta^{(0)}(G)$ is equal to

$$
\vartheta^{\prime}(G)=\max \operatorname{Tr}(J X) \text { s.t. } \operatorname{Tr}(X)=1, \quad X_{i j}=0(i j \in E), X \succeq 0, X \geq 0
$$

(see [5]). Thus, $\vartheta^{(0)}(G) \leq \vartheta(G)$, since program (7) without the nonnegativity condition is a formulation of the theta number (1).

The problem of finding a sum of squares decomposition for a polynomial of degree $2 d$ can be formulated as a semidefinite program involving matrices of size $O\left(n^{d}\right)$ and $O\left(n^{2 d}\right)$ variables (see, e.g., [16]). Therefore, for fixed $r$, program (6) can be reformulated as a semidefinite program of polynomial size and thus $\vartheta^{(r)}(G)$ can be computed in polynomial time (to any precision). 
Let us observe that, for the matrix $M:=\alpha\left(I+A_{G}\right)-J$, the polynomial $p_{M}^{(r)}(x)$ has a negative coefficient for any $r \geq 0$ when $\alpha=\alpha(G) \geq 2$. To see it, recall from [1, p. 169, Theorem 2.2] that

$$
p_{M}^{(r)}(x)=\sum_{\beta \in I(n, r+2)} \frac{r !}{\beta !} c_{\beta} x^{2 \beta}, \quad \text { where } c_{\beta}:=\beta^{\mathrm{T}} M \beta-\beta^{\mathrm{T}} \operatorname{diag}(M) .
$$

If $S(\beta)$ is a stable set, then $c_{\beta}=\alpha \sum_{i} \beta_{i}\left(\beta_{i}-1\right)-(r+1)(r+2)$. Write $r+2=q \alpha+s$ with $q, s \in \mathbb{Z}_{+}, 0 \leq s<\alpha$; then $c_{\beta}<0$ for $\beta=(q+1, \ldots, q+1, q, \ldots, q, 0, \ldots 0)$ with $s$ entries equal to $q+1, \alpha-s$ entries equal to $q$, and $S(\beta)$ being a stable set.

On the other hand the matrix $M:=\alpha(1+\epsilon)\left(I+A_{G}\right)-J$ with $\alpha=\alpha(G)$ and $\epsilon=\frac{\alpha-1}{\alpha^{2}-\alpha+1}$, belongs to the cone $\mathcal{K}_{n}^{(r)}$ for $r \geq \alpha^{2}$ since all the coefficients of the polynomial $p_{M}^{(r)}(x)$ are nonnegative [5]. This implies that $\alpha(G) \leq \vartheta^{(r)}(G) \leq$ $\alpha(G)(1+\epsilon)<\alpha(G)+1$, which gives the following result of de Klerk and Pasechnik [5]

$$
\alpha(G)=\left\lfloor\vartheta^{(r)}(G)\right\rfloor \quad \text { for } r \geq \alpha(G)^{2} .
$$

It is also shown in [5] that

$$
\vartheta^{(1)}(G) \leq 1+\max _{i \in V} \vartheta^{(0)}\left(G \backslash i^{\perp}\right) .
$$

Therefore, $\vartheta^{(1)}(G)=\alpha(G)$ when $\alpha(G) \leq 2$. More generally, de Klerk and Pasechnik [5] conjecture:

Conjecture $1 \vartheta^{(r)}(G)=\alpha(G)$ for $r \geq \alpha(G)-1$.

\subsection{Dual formulation}

Using conic duality, the bound $\vartheta^{(r)}(G)$ from (6) can be reformulated as

$$
\vartheta^{(r)}(G)=\max \operatorname{Tr}(J X) \quad \text { subject to } \operatorname{Tr}\left(\left(I+A_{G}\right) X\right)=1, X \in\left(\mathcal{K}_{n}^{(r)}\right)^{*} .
$$

As the programs (6) and (10) are strictly feasible, there is no duality gap and the optima in (6) and (10) are indeed attained [5]. For $r=0$, it follows from (5) that $\left(\mathcal{K}_{n}^{(0)}\right)^{*}$ is the cone of doubly nonnegative (i.e., positive semidefinite and nonnegative) matrices. For $r \geq 1$, one can give an explicit description of the dual cone $\left(\mathcal{K}_{n}^{(r)}\right)^{*}$. As a first step we introduce a class of matrices defined in (11).

Definition 1 Let $y=\left(y_{\delta}\right)_{\delta \in I(n, 2 r+4)}$ be given.

(i) Define the matrix ${ }^{2} N_{r+2}(y)$ indexed by $I(n, r+2)$, whose $\left(\beta, \beta^{\prime}\right)$-th entry is equal to $y_{\beta+\beta^{\prime}}$, for $\beta, \beta^{\prime} \in I(n, r+2)$.

2 Such a matrix is known as a moment matrix; for details see, e.g., Lasserre [7]. 
(ii) For $\gamma \in I(n, r), N^{\gamma}(y)$ denotes the principal submatrix of $N_{r+2}(y)$ indexed by $\gamma+2 e_{1}, \ldots, \gamma+2 e_{n}$; that is, $N^{\gamma}(y)$ is the $n \times n$ matrix with $(i, j)$-th entry $y_{2 \gamma+2 e_{i}+2 e_{j}}$, for $i, j=1, \ldots, n$.

(iii) Define the $n \times n$ matrix

$$
C(y):=\sum_{\gamma \in I(n, r)} \frac{r !}{\gamma !} N^{\gamma}(y) .
$$

\section{Definition 2 Define the cone}

$$
\mathcal{C}_{n}^{(r)}:=\left\{Z \in \mathbb{R}^{n \times n} \mid Z=C(y) \text { for some } y \in \mathbb{R}^{I(n, 2 r+4)} \text { with } N_{r+2}(y) \succeq 0\right\} .
$$

Notice that the matrix $C(y)$ in (11) involves only entries of $y$ indexed by even sequences. Therefore in the definition of the cone $\mathcal{C}_{n}^{(r)}$ one can assume w.l.o.g. that $y_{\delta}=0$ whenever $\delta$ has an odd component.

Proposition 1 The cones $\mathcal{K}_{n}^{(r)}$ and $\mathcal{C}_{n}^{(r)}$ are dual of each other; that is, $\mathcal{C}_{n}^{(r)}=\left(\mathcal{K}_{n}^{(r)}\right)^{*}$ and $\mathcal{K}_{n}^{(r)}=\left(\mathcal{C}_{n}^{(r)}\right)^{*}$.

The proof relies on a known duality relationship between the cone of sums of squares of polynomials and the cone of positive semidefinite moment matrices. Nevertheless, the explicit description of $\mathcal{C}_{n}^{(r)}$, the dual of $\mathcal{K}_{n}^{(r)}$, is new to the best of our knowledge. Given $u=\left(u_{\alpha}\right) \in \mathbb{R}^{I(n, r+2)}$, consider the polynomial $p(x):=\left(\sum_{\alpha} u_{\alpha} x^{\alpha}\right)^{2}$; then the following identity holds

$$
y^{\mathrm{T}} p=u^{\mathrm{T}} N_{r+2}(y) u \quad \text { for any } y \in \mathbb{R}^{I(n, 2 r+4)} .
$$

Indeed, $y^{\mathrm{T}} p=\sum_{\delta} y_{\delta} p_{\delta}=\sum_{\delta} y_{\delta}\left(\sum_{\alpha, \beta \mid \delta=\alpha+\beta} u_{\alpha} u_{\beta}\right)=\sum_{\alpha, \beta} u_{\alpha} u_{\beta} y_{\alpha+\beta}$, which is equal to $u^{\mathrm{T}} N_{r+2}(y) u$. Define the two cones

$$
\begin{gathered}
\Sigma_{2 r+4}:=\left\{p=\left(p_{\alpha}\right) \in \mathbb{R}^{I(n, 2 r+4)} \mid \sum_{\alpha} p_{\alpha} x^{\alpha} \text { is a sum of squares of polynomials }\right\} \\
\mathcal{N}_{2 r+4}:=\left\{y \in \mathbb{R}^{I(n, 2 r+4)} \mid N_{r+2}(y) \succeq 0\right\} .
\end{gathered}
$$

Lemma 1 The two cones $\mathcal{N}_{2 r+4}$ and $\Sigma_{2 r+4}$ are dual of each other; that is, $\mathcal{N}_{2 r+4}=$ $\left(\Sigma_{2 r+4}\right)^{*}$ and $\Sigma_{2 r+4}=\left(\mathcal{N}_{2 r+4}\right)^{*}$.

Proof The equality $\mathcal{N}_{2 r+4}=\left(\Sigma_{2 r+4}\right)^{*}$ follows as a direct application of (12) and it implies the equality $\Sigma_{2 r+4}=\left(\mathcal{N}_{2 r+4}\right)^{*}$ since $\Sigma_{2 r+4}$ is a closed cone (see [20, p. 37, Prop. 3.6]).

Proof (of Proposition 1) Let $C(y) \in \mathcal{C}_{n}^{(r)}$, let $M$ be a symmetric $n \times n$ matrix and let $p_{M}^{(r)}$ be the associated polynomial via (4). Using (8), one can verify that

$$
\operatorname{Tr}(M C(y))=y^{\mathrm{T}} p_{M}^{(r)} \quad \text { for any } y \in \mathbb{R}^{I(n, 2 r+4)} .
$$


Indeed,

$$
\begin{gathered}
\operatorname{Tr}(M C(y))=\sum_{i, j=1}^{n} M_{i j} C(y)_{i j}=\sum_{i, j=1}^{n} M_{i j} \sum_{\gamma \in I(n, r)} \frac{r !}{\gamma !} y_{2 \gamma+2 e_{i}+2 e_{j}} \\
=\sum_{\beta \in I(n, r+2)}\left(\sum_{i \mid \beta_{i} \geq 2} \frac{r !}{\left(\beta-2 e_{i}\right) !} M_{i i} y_{2 \beta}+\sum_{i \neq j \mid \beta_{i}, \beta_{j} \geq 1} \frac{r !}{\left(\beta-e_{i}-e_{j}\right) !} M_{i j} y_{2 \beta}\right) \\
=\sum_{\beta \in I(n, r+2)}\left(\sum_{i} \frac{r ! \beta_{i}\left(\beta_{i}-1\right)}{\beta !} M_{i i} y_{2 \beta}+\sum_{i \neq j} \frac{r ! \beta_{i} \beta_{j}}{\beta !} M_{i j} y_{2 \beta}\right) \\
=\sum_{\beta \in I(n, r+2)} \frac{r !}{\beta !} y_{2 \beta}\left(\beta^{\mathrm{T}} M \beta-\beta^{\mathrm{T}} \operatorname{diag}(M)\right)=y^{\mathrm{T}} p_{M}^{(r)} .
\end{gathered}
$$

Using (13) and the equality $\left(\mathcal{N}_{2 r+4}\right)^{*}=\Sigma_{2 r+4}$, one can immediately conclude that $\mathcal{K}_{n}^{(r)}=\left(\mathcal{C}_{n}^{(r)}\right)^{*}$. The cone $\mathcal{C}_{n}^{(r)}$ is closed since it consists of linear combinations of positive semidefinite matrices and the positive semidefinite cone is closed. Hence $\mathcal{C}_{n}^{(r)}=\left(\mathcal{K}_{n}^{(r)}\right)^{*}$.

Let us note for further reference the following identities which follow using (8) and (13)

$$
\begin{aligned}
\operatorname{Tr}(J C(y)) & =\sum_{\beta \in I(n, r+2)} \frac{(r+2) !}{\beta !} y_{2 \beta}, \\
\operatorname{Tr}(C(y)) & =\sum_{\beta \in I(n, r+2)} \frac{r !}{\beta !} y_{2 \beta}\left(\sum_{i=1}^{n} \beta_{i}^{2}-\beta_{i}\right) .
\end{aligned}
$$

2.4 Semidefinite relaxations of the stable set polytope

Let $P_{\text {stab }}(G)$ denote the stable set polytope of $G$, defined as the convex hull of the incidence vectors of the stable sets in $G$. For an integer $r \geq 0$, define the set

$$
\begin{array}{r}
P^{(r)}(G):=\left\{x \in \mathbb{R}^{n} \mid x=\operatorname{diag}(X) \text { for some } X \in \mathcal{C}_{n}^{(r)}\right. \text { satisfying } \\
\left.\operatorname{Tr}\left(A_{G} X\right)=0, X-x x^{\mathrm{T}} \succeq 0\right\}
\end{array}
$$

and define the parameter

$$
\tilde{\vartheta}^{(r)}(G):=\max _{x \in P^{(r)}(G)} \sum_{i \in V} x_{i} .
$$

Lemma $2 P_{\text {stab }}(G) \subseteq P^{(r)}(G)$ and $\alpha(G) \leq \tilde{\vartheta}^{(r)}(G) \leq \vartheta^{(r)}(G)$ for any integer $r \geq 0$. 
Proof Given a stable set $S$ with incidence vector $x:=\chi^{S}$, define the vector $y \in \mathbb{R}^{I(n, 2 r+4)}$ with $y_{\delta}=\frac{1}{|S|^{r}}$ if $\delta$ is even and $S(\delta) \subseteq S$, and $y_{\delta}=0$ otherwise. Then, $|S|^{r} N_{r+2}(y)$ is a $0 / 1$ block diagonal matrix, whose blocks are indexed by the sets $\mathcal{O}_{I}:=\left\{\alpha \in I(n, r+2) \mid S(\alpha) \subseteq S, S_{\text {odd }}(\alpha)=I\right\}$ for $I \subseteq S$, and the set $\mathcal{O}:=\{\alpha \in I(n, r+2) \mid S(\alpha) \nsubseteq S\}$. Each $\mathcal{O}_{I} \times \mathcal{O}_{I}$ block is the all-ones matrix, and the $\mathcal{O} \times \mathcal{O}$ block is zero. Hence $N_{r+2}(y) \succeq 0$. For $\gamma \in I(n, r)$,

$$
|S|^{r} N^{\gamma}(y)={ }_{V \backslash S}^{S}\left(\begin{array}{cc}
S & V \backslash S \\
0 & 0 \\
0 & 0
\end{array}\right)=x x^{\mathrm{T}}
$$

if $S(\gamma) \subseteq S$, and $N^{\gamma}(y)=0$ otherwise. Hence $C(y)=\sum_{\gamma \in I(n, r)} \frac{r !}{\gamma !} N^{\gamma}(y)=$ $\sum_{\gamma \in I(n, r)} \frac{r !}{\gamma !} \frac{1}{|S|^{r}} x x^{\mathrm{T}}=x x^{\mathrm{T}}$. Setting $X:=C(y)=x x^{\mathrm{T}}$, we have $\operatorname{Tr}\left(A_{G} X\right)=0$, and $x=\operatorname{diag}(X)$, which shows that $x \in P^{(r)}(G)$. This shows the inclusion: $P_{\text {stab }}(G) \subseteq P^{(r)}(G)$ which in turn implies the inequality: $\alpha(G) \leq \tilde{\vartheta}^{(r)}(G)$. The inequality: $\tilde{\vartheta}^{(r)}(G) \leq \vartheta^{(r)}(G)$ follows from Lemma 3 below.

The sets $P^{(r)}(G)$ provide a hierarchy of semidefinite relaxations for $P_{\text {stab }}(G)$. It is known that $P_{\text {stab }}(G)=P^{(0)}(G)$ when $G$ is a perfect graph (see [6]). A natural question to ask is whether the analogue of Conjecture 1 may hold, asserting that $P_{\text {stab }}(G)=P^{(r)}(G)$ for $r \geq \alpha(G)-1$. We are able to give a positive answer only in the case $r=1$; see Corollary 2 below.

For this, given positive node weights $w \in \mathbb{R}_{+}^{V}$, we have to compare the weighted stability number $\alpha_{w}(G):=\max _{x \in P_{\text {stab }}(G)} w^{\mathrm{T}} x$ and the weighted parameter

$$
\tilde{\vartheta}_{w}^{(r)}(G):=\max _{x \in P^{(r)}(G)} w^{\mathrm{T}} x .
$$

Busygin [3] shows the following extension to the weighted case of the Motzkin-Straus theorem.

Theorem 1 [3] Given $w_{i}>0(i \in V)$, set $w_{\min }:=\min _{i \in V} w_{i}$. Then,

$$
\frac{w_{\min }}{\alpha_{w}(G)}=\min _{x \in \Delta} x^{\mathrm{T}}\left(\operatorname{Diag}\left(\frac{w_{\min }}{w}\right)+A_{G}\right) x .
$$

In other words, the matrix $\alpha_{w}(G)\left(\operatorname{Diag}\left(\frac{1}{w}\right)+\frac{1}{w_{\min }} A_{G}\right)-J$ is copositive or, equivalently, the matrix $\alpha_{w}(G)\left(\operatorname{Diag}(w)+A_{G, w}\right)-w w^{\mathrm{T}}$ is copositive, where $A_{G, w}$ is the matrix whose $i j$-th entry is $\frac{w_{i} w_{j}}{w_{\min }}$ if $i j \in E$ and 0 otherwise. Set

$$
w_{\max }:=\max _{i \in V} w_{i}, \quad W_{G}:=\frac{\left(w_{\max }\right)^{2}}{w_{\min }} .
$$

The matrix $\alpha_{w}(G)\left(\operatorname{Diag}(w)+W_{G} A_{G}\right)-w w^{\mathrm{T}}$ is also copositive, since the entries of $A_{G, w}$ are at most $W_{G}$. This leads us to define the following weighted analogue of the parameter $\vartheta^{(r)}(\cdot)$ : 


$$
\vartheta_{w}^{(r)}(G):=\min t \quad \text { subject to } t\left(\operatorname{Diag}(w)+W_{G} A_{G}\right)-w w^{T} \in \mathcal{K}_{n}^{(r)} .
$$

This definition reduces to the original definition (6) when all weights are equal to 1 .

Lemma 3 The parameters (17) and (19) satisfy: $\tilde{\vartheta}_{w}^{(r)}(G) \leq \vartheta_{w}^{(r)}(G)$.

Proof Assume $M:=t\left(\operatorname{Diag}(w)+W_{G} A_{G}\right)-w w^{\mathrm{T}} \in \mathcal{K}_{n}^{(r)}$ and let $x=\operatorname{diag}(X)$ where $X \in \mathcal{C}_{n}^{(r)}, \operatorname{Tr}\left(A_{G} X\right)=0, X-x x^{\mathrm{T}} \succeq 0$. Then, $0 \leq \operatorname{Tr}(M X)=t w^{\mathrm{T}} x-w^{\mathrm{T}} X w$, yielding $t w^{\mathrm{T}} x \geq w^{\mathrm{T}} X w \geq\left(w^{\mathrm{T}} x\right)^{2}$ and thus $t \geq w^{\mathrm{T}} x$. This gives the desired inequality.

Lemma 4 For $r=0, \tilde{\vartheta}_{w}^{(0)}(G)=\vartheta_{w}^{(0)}(G)$. Therefore, $\vartheta_{w}^{(0)}(G)=\alpha_{w}(G)$ when $G$ is a perfect graph.

Proof It remains to show the inequality: $\vartheta_{w}^{(0)}(G) \leq \tilde{\vartheta}_{w}^{(0)}(G)$. For this, we first observe that

$$
\vartheta_{w}^{(0)}(G) \leq \phi(G):=\min t \quad \text { subject to } t \operatorname{Diag}(w)+y A_{G}-w w^{T} \in \mathcal{K}_{n}^{(0)} .
$$

Our argument is similar to the one used by de Klerk and Pasechnik [5] in the unweighted case. Assume $M:=t \operatorname{Diag}(w)+y A_{G}-w w^{\mathrm{T}} \in \mathcal{K}_{n}^{(0)}$. Then, $M=P+N$, where $P \geq 0, N \geq 0, \operatorname{diag}(N)=0$. Hence, $t\left(\operatorname{Diag}(w)+W_{G} A_{G}\right)-w w^{\mathrm{T}}=$ $M+\left(t W_{G}-y\right) A_{G}=P+N+\left(t W_{G}-y\right) A_{G}$. It suffices now to verify that $N^{\prime}:=N+\left(t W_{G}-y\right) A_{G} \geq 0$. For this pick an edge, say $12 \in E$. As $P \succeq 0$, we have $P_{11}+P_{22} \geq 2 P_{12}$, yielding $t\left(w_{1}+w_{2}\right)-2\left(y-N_{12}\right) \geq\left(w_{1}-w_{2}\right)^{2}$. Finally, $2 N_{12}^{\prime}=2 N_{12}+2 t \frac{w_{\max }^{2}}{w_{\min }}-2 y \geq t\left(w_{1}+w_{2}\right)-2\left(y-N_{12}\right) \geq 0$ proves $(20)$.

Next, using conic duality, we obtain that $\phi(G)=\max w^{\mathrm{T}} X w \quad$ subject to $\operatorname{Tr}(\operatorname{Diag}(w) X)=1, \operatorname{Tr}\left(A_{G} X\right)=0, X \in \mathcal{C}_{n}^{(0)}$.

Set $u:=\left(\sqrt{w_{i}}\right)_{i=1}^{n}$. Rescaling $X$ by $Y=\operatorname{Diag}(u) X \operatorname{Diag}(u)$, we find that

$$
\phi(G)=\max u^{\mathrm{T}} Y u \quad \text { subject to } \operatorname{Tr}(Y)=1, \operatorname{Tr}\left(A_{G} Y\right)=0, Y \in \mathcal{C}_{n}^{(0)} .
$$

(As $\mathcal{C}_{n}^{(0)}$ consists of the nonnegative positive semidefinite matrices, it is closed under the above rescaling.) We can now conclude that $\phi(G) \leq \tilde{\vartheta}_{w}^{(0)}(G)$; this is the same proof as for Theorem 67.11 in [22] (which gives the result with the cone $\mathcal{C}_{n}^{(0)}$ being replaced by the cone of positive semidefinite matrices).

\subsection{The main results}

In this paper we prove the following results. 
Theorem 2 For a graph $G$ and a positive integer $r \leq \min (\alpha(G)-1,6)$,

$$
\vartheta^{(r)}(G) \leq r+\max _{S \subseteq V \text { stable, }|S|=r} \vartheta^{(0)}\left(G \backslash S^{\perp}\right),
$$

Moreover, (21) holds for $r=7=\alpha(G)-1$.

Corollary 1 Conjecture 1 holds for $\alpha(G) \leq 8$; that is,

$$
\vartheta^{(\alpha(G)-1)}(G)=\alpha(G) \quad \text { if } \alpha(G) \leq 8 .
$$

Theorem 3 For a graph $G$ with positive node weights $w \in \mathbb{R}^{V}$,

$$
\vartheta_{w}^{(1)}(G) \leq \max _{i \in V}\left(w_{i}+\vartheta_{w}^{(0)}\left(G \backslash i^{\perp}\right)\right) .
$$

Corollary $2 P_{\text {stab }}(G)=P^{(1)}(G)$ if $G \backslash i^{\perp}$ is perfect for all $i \in V$; this holds in particular if $\alpha(G)=2$.

Theorem 4 For $r \geq 1$, the parameters from (2), (10) and (16) satisfy

$$
\operatorname{las}^{(r)}(G) \leq \tilde{\vartheta}^{(r-1)}(G) \leq \vartheta^{(r-1)}(G) .
$$

Corollary 1 follows directly from Theorem 2. Analogously, Corollary 2 follows from Theorem 3 together with Lemma 4. The proofs for Theorems 2, 3, 4 are given in Sects. 3, 4, 5, respectively.

Our proof technique for Theorem 2 does not apply to the case when $\alpha(G) \geq 9$. It is quite more complicated than the proof of convergence in $\alpha(G)$ steps for the Lovász-Schrijver and the Lasserre semidefinite hierarchies. One of the main difficulties (as pointed out later in the proof) comes from the fact that, for $r \geq 1$, the cone $\mathcal{K}_{n}^{(r)}$ is not invariant under some simple matrix operations, like extending a matrix by adding a zero row and column to it, or rescaling it by positive multipliers (which obviously preserve copositivity and positive semidefiniteness). For instance, when $G$ is a circuit of length 5, the matrix $M:=2\left(I+A_{G}\right)-J$ belongs to $\mathcal{K}_{5}^{(1)}$, but adding a zero row and column yields a matrix that does not belong to $\mathcal{K}_{6}^{(1)}$. We thank E. de Klerk for communicating this example to us.

As Theorem 4 shows, the bound las ${ }^{(r)}(G)$ is at least as good as $\tilde{\vartheta}^{(r-1)}(G)$. There exist in fact graphs for which strict inequality: $\operatorname{las}^{(2)}(G)<\tilde{\vartheta}^{(1)}(G)$ holds. For this, given integers $2 \leq d \leq n$, consider the graph $G(n, d)$ with node set $\mathcal{P}(V)$ $(|V|=n)$ where $I, J \in \mathcal{P}(V)$ are connected by an edge if $|I \Delta J| \in\{1, \ldots, d-1\}$. Then $\alpha(G(n, d))$ is the maximum cardinality of a binary code of word length $n$ with minimum distance $d$. Delsarte [4] introduced a linear programming bound which coincides with the parameter $\vartheta^{\prime}(G(n, d))$ ([21]). Schrijver [23] introduced a stronger semidefinite bound which roughly ${ }^{3}$ lies between the

\footnotetext{
3 Indeed, the formulation of Schrijver's bound has an additional constraint, namely, $x_{i j k} \leq x_{i j}$ for all $i, j, k \in V$, which does not appear in the definition of the bound las ${ }^{(r)}(G)$ used in the present paper.
} 
bounds las ${ }^{(1)}(G(n, d))$ and las ${ }^{(2)}(G(n, d))([10])$. While $G(n, d)$ has $2^{n}$ vertices, Schrijver's bound can be computed via a semidefinite program of size $O\left(n^{3}\right)$ (using a block diagonalization of the underlying Terwiliger algebra). It turns out that the same algebraic property holds for the bound $\vartheta^{(1)}(G(n, d))$; thus we could compute this bound as well as Schrijver's bound for the parameters $(n, d)=(17,4),(17,6),(17,8)$, and we found

$$
\begin{gathered}
\operatorname{las}^{(2)}(G(17,4)) \leq 3276<3607 \leq \vartheta^{(1)}(G(17,4)) \\
\operatorname{las}^{(2)}(G(17,6)) \leq 352<395 \leq \vartheta^{(1)}(G(17,6)) \\
\operatorname{las}^{(2)}(G(17,8)) \leq 41<42 \leq \vartheta^{(1)}(G(17,8)) .
\end{gathered}
$$

\section{Proof of Theorem 2}

Let $G=(V, E)$ be a graph with stability number $\alpha(G), V=\{1, \ldots, n\}$ and $1 \leq r \leq \alpha(G)-1$ an integer. Set

$$
t:=r+\max _{S \subseteq V \text { stable, }|S|=r} \vartheta^{(0)}\left(G \backslash S^{\perp}\right) .
$$

Then, $t \geq r+1$. As $t-r \geq \vartheta^{(0)}\left(G \backslash S^{\perp}\right)$, we deduce that

$$
(t-r)\left(I+A_{G \backslash S^{\perp}}\right)-J \in \mathcal{K}_{n-\left|S^{\perp}\right|}^{(0)} \quad \text { for any stable set } S \text { in } G \text { of size } r .
$$

In order to prove Theorem 2 , we have to show that, for $1 \leq r \leq \min (\alpha(G)-1,6)$,

$$
M:=t\left(I+A_{G}\right)-J \in \mathcal{K}_{n}^{(r)} .
$$

We need some notation. Let $B$ be an $m \times n$ matrix. We say that $B$ is a $q \times s$ block matrix if the set $\{1, \ldots, m\}$ indexing its rows can be partitioned into $Q_{1} \cup \ldots \cup Q_{q}$ and the set $\{1, \ldots, n\}$ indexing its columns can be partitioned into $S_{1} \cup \ldots \cup S_{s}$ in such a way that, for any $h \in\{1, \ldots, q\}, h^{\prime} \in\{1, \ldots, s\}$, the entries $B_{i j}$ for $i \in Q_{h}, j \in S_{h^{\prime}}$ are all equal to the same value, say $\tilde{b}_{h h^{\prime}}$. In other words, $B$ is obtained from the matrix $\tilde{B}:=\left(\tilde{b}_{h h^{\prime}}\right)_{h \in\{1, \ldots, q\}}$ by suitably duplicating rows and columns. We call $\tilde{B}$ the skeleton of the block matrix $B$. Obviously, $B \succeq 0$ if and only if $\tilde{B} \succeq 0$ (assuming $m=n, q=s$ ); moreover, $B \in \mathcal{K}_{n}^{(r)}$ if and only if $\tilde{B} \in \mathcal{K}_{q}^{(r)}$ (see Lemma 15 below).

Finally, for $x \in \mathbb{R}^{n}$, set $v(x):=\left(x_{i}^{2}\right)_{i=1}^{n}$.

The following observation plays a central role in the proof.

Lemma 5 Let $X(i)(i \in V)$ be symmetric matrices satisfying the condition

$$
X(i)_{j k}+X(j)_{i k}+X(k)_{i j} \geq 0 \quad \text { for all } i, j, k \in V,
$$

then the polynomial $\sum_{i \in V} x_{i}^{2} v(x)^{\mathrm{T}} X(i) v(x)=\sum_{i, j, k \in V} x_{i}^{2} x_{j}^{2} x_{k}^{2} X(i)_{j k}$ is a sum of squares. 
Proof The polynomial $\sum_{i, j, k \in V} x_{i}^{2} x_{j}^{2} x_{k}^{2} X(i)_{j k}$ is equal to

$$
\begin{gathered}
\sum_{\substack{i, j, k) \in V^{3} \\
i \neq j \neq k \neq i}} x_{i}^{2} x_{j}^{2} x_{k}^{2}\left[X(i)_{j k}+X(i)_{j k}+X(i)_{j k}\right] \\
+\sum_{\substack{(i, j) \in V^{2} \\
i \neq j}} x_{i}^{2} x_{j}^{4}\left[X(i)_{j j}+2 X(j)_{i j}\right]+\sum_{i \in V} x_{i}^{6} X(i)_{i i},
\end{gathered}
$$

which is a sum of squares, since all coefficients are nonnegative by (26).

Our strategy will be to construct matrices $X\left(\left\{i_{1}, \ldots, i_{k}\right\}, i\right)(i \in V)$ satisfying (26) when $\left\{i_{1}, \ldots, i_{k}\right\}$ is a stable set of size $k \leq r$. We will use them to recursively decompose $M$ into $M-X\left(i_{1}\right)-X\left(i_{1}, i_{2}\right)-\cdots-X\left(i_{1}, \ldots, i_{k}\right)$ in such way that at the last level $k=r$ we obtain matrices in $\mathcal{K}_{n}^{(0)}$. It turns out that this last property holds for $r \leq 7$, but not for $r=8$. This is why we are able to prove the conjecture only for graphs with the stability number at most 8 .

3.1 Defining sets of matrices satisfying the linear condition (26)

Let $S$ be a stable set of cardinality $k, 0 \leq k \leq r$. We define a set of matrices $X(S, i)$ (for $i \in V$ ) indexed by $V$ that satisfy the condition (26). Set $m_{0}:=1$ and $m_{k}:=\frac{t^{k}}{(t-1) \cdots(t-k)}$ for $k=1, \ldots, r$. (Recall that $t \geq r+1>k$.)

For $i \in S^{\perp}, X(S, i)$ is the symmetric matrix whose entry at position $(u, v)$ is defined as follows:

$$
m_{k} \text { times } \begin{cases}0 & \text { if } u \text { or } v \in S^{\perp} \\ t-k-1 & \text { if } u, v \in V \backslash S^{\perp} \text { and } u \simeq v \\ -1 & \text { if } u, v \in V \backslash S^{\perp} \text { and } u \neq v\end{cases}
$$

For $i \notin S^{\perp}, X(S, i)$ is the symmetric matrix whose entry at position $(u, v)$ is defined as follows:

$$
m_{k} \text { times } \begin{cases}0 & \text { if } u, v \in S^{\perp} \\ -\frac{t-k-1}{2} & \text { if } u \in S^{\perp}, v \in i^{\perp} \backslash S^{\perp} \\ \frac{1}{2} & \text { if } u \in S^{\perp}, v \in V \backslash\left(S^{\perp} \cup i^{\perp}\right) \\ 0 & \text { if } u, v \in i^{\perp} \backslash S^{\perp} \text { and } u \simeq v \\ -(t-k) & \text { if } u, v \in i^{\perp} \backslash S^{\perp} \text { and } u \neq v \\ t-\frac{k}{2} & \text { if } u \in i^{\perp} \backslash S^{\perp}, v \in V \backslash\left(S^{\perp} \cup i^{\perp}\right) \text { and } u \simeq v \\ \frac{k}{2} & \text { if } u \in i^{\perp} \backslash S^{\perp}, v \in V \backslash\left(S^{\perp} \cup i^{\perp}\right) \text { and } u \neq v \\ -k & \text { if } u, v \in V \backslash\left(S^{\perp} \cup i^{\perp}\right) \text { and } u \simeq v \\ 0 & \text { if } u, v \in V \backslash\left(S^{\perp} \cup i^{\perp}\right) \text { and } u \neq v .\end{cases}
$$

If $S=\left\{i_{1}, \ldots, i_{k}\right\}$, we also denote $X(S, i)$ as $X\left(i_{1}, \ldots, i_{k}, i\right)$. When $S=\emptyset$, we set $X(\emptyset, i)=: X(i)$. Given an ordering $(S)=\left(i_{1}, \ldots, i_{k}\right)$ of the elements of $S$, define the matrix

$$
M((S)):=M-X\left(i_{1}\right)-X\left(i_{1}, i_{2}\right)-\cdots-X\left(i_{1}, \ldots, i_{k}\right) .
$$


Lemma 6 Given a stable set $S$ of size $0 \leq k \leq r$, the matrices $X(S, i)(i \in V)$ satisfy (26).

Proof We prove $X(S, i)_{j k}+X(S, j)_{k i}+X(S, k)_{i j} \geq 0$ for all possible combinations of $i, j$ and $k$. Indeed, omitting the scalar factor $m_{k}$, we find

- For $i, j, k \in S^{\perp}: 0+0+0=0$;

- For $i, j \in S^{\perp}, k \notin S^{\perp}: 0+0+0=0$;

- For $i \in S^{\perp}, j, k \notin S^{\perp}, j \simeq k:(t-k-1)+\left(-\frac{t-k-1}{2}\right)+\left(-\frac{t-k-1}{2}\right)=0$;

- For $i \in S^{\perp}, j, k \notin S^{\perp}, j \neq k:-1+\frac{1}{2}+\frac{1}{2}=0$;

- For $i, j, k \notin S^{\perp}, i \simeq j \simeq k \simeq i: 0+0+0=0$;

- For $i, j, k \notin S^{\perp}, i \simeq j \simeq k \neq i:\left(t-\frac{k}{2}\right)+(-t+k)+\left(t-\frac{k}{2}\right)=t$;

- For $i, j, k \notin S^{\perp}, k \not \varkappa i \simeq j \not z k: \frac{k}{2}+\frac{k}{2}-k=0$;

- For $i, j, k \notin S^{\perp}, i \neq j \not z k \not i: 0+0+0=0$.

3.2 The role of the matrices $X(S, i)$ and $M((S))$ in the proof

Our objective is to prove that the matrix $M$ from (25) belongs to the cone $\mathcal{K}_{n}^{(r)}$, i.e., that the polynomial $p_{M}^{(r)}(x)=\sigma(x)^{r} v(x)^{\mathrm{T}} M v(x)$ is a sum of squares, setting $\sigma(x):=\sum_{i=1}^{n} x_{i}^{2}$. Recall that $v(x)=\left(x_{i}^{2}\right)_{i=1}^{n}$. The basic idea is to decompose $p_{M}^{(r)}(x)$ as

$$
\sigma(x)^{r-1} \sum_{i=1}^{n} x_{i}^{2} v(x)^{\mathrm{T}} X(i) v(x)+\sigma(x)^{r-1} \sum_{i=1}^{n} x_{i}^{2} v(x)^{\mathrm{T}} M((i)) v(x) .
$$

The first sum is a sum of squares by Lemmas 5 and 6 . Each matrix $M((i))$ can be written as

$$
\begin{gathered}
M((i))=M-X(i)=\begin{array}{l}
i^{\perp} \\
V \backslash i^{\perp}
\end{array}\left(\begin{array}{cc}
i^{\perp} & V \backslash i^{\perp} \\
-J & -J) J
\end{array}\right) \\
=\frac{t}{t-1}\left(\begin{array}{c}
0 \\
0 \\
0(t-1)\left(I+A_{G \backslash i^{\perp}}\right)-J
\end{array}\right) \\
\left.\begin{array}{cc}
\left.{ }^{\perp}\right)-J
\end{array}\right)+\left(\begin{array}{cc}
(t-1) J & -J \\
-J & \frac{1}{t-1} J
\end{array}\right) .
\end{gathered}
$$

When $r=1$, (29), (30) together with assumption (24) imply that $M-X(i) \in \mathcal{K}_{n}^{(0)}$ and thus $p_{M}^{(1)}(x)$ is a sum of squares; therefore (9) holds. Assume now $r \geq 2$. The last matrix in (30) is positive semidefinite. Suppose our assumption would be that $(t-1)\left(I-A_{G \backslash i^{\perp}}\right)-J \in \mathcal{K}_{n-\left|i^{\perp}\right|}^{(r-1)}$, then it would be tempting to conclude from (29) and (30) that $M-X(i) \in \mathcal{K}_{n}^{(r-1)}$ (which would then imply that $M \in \mathcal{K}_{n}^{(r)}$ and thus conclude the proof). This would be correct if we would work with cones of matrices which are closed under adding a zero row and column, but this is not 
the case for the cones $\mathcal{K}^{(r)}$ and thus this argument does not work. To go around this difficulty, we proceed as follows. If a vertex $i$ is adjacent to all other vertices, then $M((i))=(t-1) J \succeq 0$ implying that $\sigma(x)^{r-1} x_{i}^{2} v(x)^{\mathrm{T}} M((i)) v(x)$ is sum of squares. Otherwise, we further decompose the second sum in (28) by developing summands $\sigma(x)^{r-1} x_{i}^{2} v(x)^{\mathrm{T}} M((i)) v(x)$ as $\sigma(x)^{r-2} \sum_{j=1}^{n} x_{i}^{2} x_{j}^{2} v(x)^{\mathrm{T}} M((i)) v(x)$ and then as $\sigma(x)^{r-2} \sum_{j=1}^{n} x_{i}^{2} x_{j}^{2} v(x)^{\mathrm{T}} X(i, j) v(x)+\sigma(x)^{r-2} \sum_{j=1}^{n} x_{i}^{2} x_{j}^{2} v(x)^{\mathrm{T}} M((i, j)) v(x)$ (recall the def. of $M((i, j))$ from (27)). In the next step we then decompose the summands $\sigma(x)^{r-2} x_{i}^{2} x_{j}^{2} v(x)^{\mathrm{T}} M((i, j)) v(x)$ if $i^{\perp} \cup j^{\perp} \neq V$, etc. Generally, we have the following 'inclusion-exclusion' formula for the matrix $\sigma(x)^{r} M$ :

$$
\begin{aligned}
& \sigma(x)^{r} M=\sum_{h=1}^{r} \sigma(x)^{r-h} \sum_{\substack{i_{1} \in V, i_{2} \notin i_{1}^{\perp}, \ldots, i_{h-1} \notin i_{1} \cup \ldots \cup \ldots i \frac{\perp}{i_{h} \in V} \\
i_{h} \in V}} x_{i_{1}}^{2} \cdots x_{i_{h}}^{2} X\left(i_{1}, \ldots, i_{h}\right) \\
& +\sum_{h=2}^{r} \sigma(x)^{r-h} \sum_{i_{1} \in V, i_{2} \notin i_{1}^{\perp}, \ldots, i_{h-1} \notin i_{1}^{\perp} \cup \ldots \cup i_{h-2}^{\perp}} x_{i_{1}}^{2} \cdots x_{i_{h}}^{2} M\left(\left(i_{1}, \ldots, i_{h}\right)\right) \\
& +\sum_{\substack{i_{1} \in V, i_{2} \notin i_{1}^{\perp}, \ldots, i_{r-1} \notin i i_{1}^{\perp} \cup \ldots \cup i_{r-2}^{\perp} \\
i_{r} \notin i_{1} \cup \ldots \cup i i_{r-1}^{\perp}}}^{i_{h} \in i_{1}^{\perp} \cup \ldots \cup i i_{h-1}^{\perp}} x_{i_{1}}^{2} \cdots x_{i_{r}}^{2} M\left(\left(i_{1}, \ldots, i_{r}\right)\right) .
\end{aligned}
$$

Therefore, in order to show that $M \in \mathcal{K}_{n}^{(r)}$, it suffices to show that

$$
\begin{aligned}
M\left(\left(i_{1}, \ldots, i_{k}, i_{k+1}\right)\right) & \in \mathcal{K}_{n}^{(0)} \quad \text { for } S:=\left\{i_{1}, \ldots, i_{k}\right\} \text { stable, } \\
i_{k+1} & \in S^{\perp}, 1 \leq k \leq r-1,
\end{aligned}
$$

and

$$
M\left(\left(i_{1}, \ldots, i_{r}\right)\right) \in \mathcal{K}_{n}^{(0)} \quad \text { for }\left\{i_{1}, \ldots, i_{r}\right\} \text { stable. }
$$

For this we need to study the structure of the matrices $M((S))$.

\subsection{The structure of the matrices $M((S))$}

Given an ordered stable set $(S)=\left(i_{1}, i_{2}, \ldots, i_{k}\right)$ with $k=1, \ldots, r$, consider the matrix $M((S))$ from (27) and write

$$
M((S)):=\begin{array}{cc}
S^{\perp} & V \backslash S^{\perp} \\
V \backslash S^{\perp} & \left(\begin{array}{cc}
C_{k}(S) & D_{k}(S) \\
D_{k}(S)^{\mathrm{T}} & E_{k}(S)
\end{array}\right) .
\end{array}
$$

Lemma 7 The matrix $M((S))$ from (34) has the following properties.

(i) $C_{k}(S)$ is a $k \times k$ block matrix whose rows and columns are indexed by the partition of $S^{\perp}$ into $i_{1}^{\perp} \cup\left(i_{2}^{\perp} \backslash i_{1}^{\perp}\right) \cup \ldots \cup\left(i_{k}^{\perp} \backslash\left\{i_{1}, \ldots, i_{k-1}\right\}^{\perp}\right)$. Let $C_{k}$ be 
the skeleton of $C_{k}(S)\left(C_{k}\right.$ is a $k \times k$ matrix $)$ and set $d_{k}:=C_{k} e \in \mathbb{R}^{k}$. Then, $e^{\mathrm{T}} C_{k} e=\sum_{h=1}^{k} d_{k}(h)=\left(m_{k}-1\right)(t-k)^{2}$.

(ii) The matrix $D_{k}(S)$ is a $k \times 1$ block matrix, with the same partition as above for the set $S^{\perp}$ indexing its rows. Given $h \in\{1, \ldots, k\}$, all entries in the $(h, 1)$-block take the same value, which is equal to $-\frac{d_{k}(h)}{t-k}$.

(iii) For $u, v \in V \backslash S^{\perp}$, the $(u, v)$-th entry of $E_{k}(S)$ is equal to tm $_{k-1}-1$ if $u \simeq v$ and to -1 if $u \not v v$.

Proof The block structure of the matrices $C_{k}$ and $D_{k}$ is determined by the construction of the matrix $M((S))$ in $(27)$ and the shape of the matrices $X(\cdot)$ defined in Sect. 3.1. We show the lemma by induction on $k \geq 1$. For $k=1$, the matrix $M((S))=M-X\left(i_{1}\right)$ has the shape given in (29) and the desired properties hold. Assume (i), (ii), (iii) hold for a stable set $S$ of size $k \geq 1$. Let $i \in V \backslash S^{\perp}$. We show that (i), (ii), (iii) hold for the stable set $S \cup\{i\}$. Let $D_{k}^{\prime}(S)$ (resp., $D_{k}^{\prime \prime}(S)$ ) be the submatrices of $D_{k}(S)$ whose columns are indexed by $i^{\perp} \backslash S$ (resp., $V \backslash\left(S \cup i^{\perp}\right)$ ) and with the same row indices as $D_{k}(S)$. Then $C_{k+1}(S, i)$ and $D_{k+1}(S, i)$ have the following block structure:

$$
\begin{gathered}
C_{k+1}(S, i)=\left(\begin{array}{cc}
C_{k}(S) & D_{k}^{\prime}(S)+\frac{t-k-1}{2} m_{k} J \\
D_{k}^{\prime}(S)^{\mathrm{T}}+\frac{t-k-1}{2} m_{k} J^{\mathrm{T}} & \left(t m_{k-1}-1\right) J
\end{array}\right) \\
D_{k+1}(S, i)=\left(\begin{array}{c}
D_{k}^{\prime \prime}(S)-\frac{1}{2} m_{k} J \\
\left(-1-m_{k} \frac{k}{2}\right) J
\end{array}\right),
\end{gathered}
$$

where $J$ denotes the all-ones matrix of the appropriate size. By the induction assumption, the entries in the $h$-th row of $D_{k}^{\prime}(S)$ and $D_{k}^{\prime \prime}(S)$ are equal to $-\frac{d_{k}(h)}{t-k}$ (for $h=1, \ldots, k)$, and $e^{\mathrm{T}} d_{k}=e^{\mathrm{T}} C_{k} e=\left(m_{k}-1\right)(t-k)^{2}$.

We first show that $C_{k+1}(S, i)$ satisfies (i). Indeed,

$$
\begin{gathered}
e^{\mathrm{T}} C_{k+1} e=e^{\mathrm{T}} C_{k} e+2\left(-\frac{e^{\mathrm{T}} d_{k}}{t-k}+m_{k} \frac{t-k-1}{2}\right)+t m_{k-1}-1 \\
=\left(m_{k}-1\right)(t-k)^{2}-2\left(m_{k}-1\right)(t-k)+m_{k}(t-k-1) k+m_{k}(t-k)-1 \\
=m_{k}(t-k-1) t-(t-k-1)^{2}=\left(m_{k+1}-1\right)(t-k-1)^{2} .
\end{gathered}
$$

We now show that $D_{k+1}(S, i)$ satisfies (ii). Setting $d_{k+1}:=C_{k+1} e$, for $h=$ $1, \ldots, k$, we have

$$
d_{k+1}(h)=d_{k}(h)-\frac{d_{k}(h)}{t-k}+\frac{t-k-1}{2} m_{k}=(t-k-1)\left(\frac{d_{k}(h)}{t-k}+\frac{m_{k}}{2}\right) .
$$

This yields: $-\frac{d_{k+1}(h)}{t-k-1}=-\frac{d_{k}(h)}{t-k}-\frac{m_{k}}{2}$, which is indeed equal to the entries of $D_{k+1}(S, i)$ in its $h$-th row. The entries of $D_{k+1}(S, i)$ in its $(k+1)$-th row are equal 
to $-1-m_{k} \frac{k}{2}$, thus equal to $-\frac{d_{k+1}(k+1)}{t-k-1}$ since

$$
\begin{gathered}
d_{k+1}(k+1)=\sum_{h=1}^{k}\left(-\frac{d_{k}(h)}{t-k}+\frac{k(t-k-1)}{2} m_{k}\right)+t m_{k-1}-1 \\
=-\frac{e^{\mathrm{T}} C_{k} e}{t-k}+k \frac{t-k-1}{2} m_{k}+t m_{k-1}-1=(t-k-1)\left(1+\frac{k m_{k}}{2}\right) .
\end{gathered}
$$

We finally show that $E_{k+1}(S, i)$ satisfies (iii). Indeed, its $(u, v)$-th entry remains the same as in $E_{k}(S)$, i.e., equal to -1 , if $u \not 千 v$ and, for $u \simeq v$, it is equal to $t m_{k-1}-1+k m_{k}=(t-k) m_{k}-1+k m_{k}=t m_{k}-1$.

Corollary 3 Let $S$ be a stable set of size $k=1, \ldots, r$. Then,

$$
\begin{gathered}
G((S)):=\left(\begin{array}{cc}
C_{k}(S) & D_{k}(S) \\
D_{k}(S)^{\mathrm{T}} & \left(m_{k}-1\right) J
\end{array}\right) \succeq 0 \Longleftrightarrow C_{k}(S) \succeq 0, \\
M((S))=G((S))+m_{k}\left(\begin{array}{lc}
0 & 0 \\
0(t-k)\left(I+A_{G \backslash S^{\perp}}\right)-J
\end{array}\right), \\
M((S, i))=G((S)) \quad \text { if } i \in S^{\perp} .
\end{gathered}
$$

Proof By Lemma 7, $C_{k}(S), D_{k}(S)$ are block matrices; hence $G((S)) \succeq 0$ if and only if its skeleton $G:=\left(\begin{array}{cc}C_{k} & -\frac{1}{t-k} C_{k} e \\ -\frac{1}{t-k} e^{\mathrm{T}} C_{k} & m_{k}-1\end{array}\right)$ is positive semidefinite. Now, $G \succeq 0 \Longleftrightarrow C_{k} \succeq 0$ since the last column of $G$ is a linear combination of the first $k$ columns; thus (37) holds. Relations (38), (39) follow using the definitions.

Therefore, (32), (33) hold (and thus $M \in \mathcal{K}_{n}^{(r)}$ ) if we can show that $C_{k}(S) \succeq 0$ for any stable set $S$ of size $k \leq r$. As $C_{k}(S)$ is a block matrix, it suffices to show that its skeleton $C_{k}$ is positive semidefinite. Moreover, it suffices to show that $C_{r} \succeq 0$ since, in view of (35), the matrices $C_{k}(1 \leq k \leq r)$ are in fact the leading principal submatrices of $C_{r}$.

\subsection{The matrix $C_{r}$ is positive semidefinite for $r \leq \min (\alpha(G)-1,6)$}

Recall that the entries of $C_{r}$ depend on the parameter $t$; thus one may alternatively write $C_{r}$ as $C_{r}(t)$. Our task is now to show that $C_{r}(t) \succeq 0$ for all $t \geq r+1$ and $r \leq \min (\alpha(G)-1,6)$. We achieve this by proving that

$$
\operatorname{det} C_{k}(t)>0 \quad \text { for } t \geq r+1, k=1, \ldots, r \text {. }
$$

The proof of (40) relies on establishing a recurrence relationship among the determinants of $C_{k}(t)$. We need the following lemma.

Lemma 8 Assume $C_{k+1}$ is nonsingular for $k \geq 1$. Then,

$$
e^{\mathrm{T}}\left(C_{k+1}\right)^{-1} e=\frac{t^{2}}{(t-k)^{2}} \frac{\operatorname{det} C_{k}}{\operatorname{det} C_{k+1}} .
$$


Proof Write $C_{k+1}:=\left(\begin{array}{cc}C_{k} & x \\ x^{\mathrm{T}} & a\end{array}\right),\left(C_{k+1}\right)^{-1}:=\left(\begin{array}{cc}A & y \\ y^{\mathrm{T}} & b\end{array}\right)$. Then,
(a) $A C_{k}+y x^{\mathrm{T}}=I$;
(b) $C_{k} y+b x=0$;
(c) $A x+a y=0$;
(d) $x^{\mathrm{T}} y+a b=1$.

By Lemma 7 and (35), $a=t m_{k-1}-1=(t-k) m_{k}-1$ and $x=\rho_{k} e-\frac{1}{t-k} C_{k} e$, setting $\rho_{k}:=m_{k} \frac{t-k-1}{2}$. Moreover, $e^{\mathrm{T}} C_{k} e=\left(m_{k}-1\right)(t-k)^{2}$, implying

$$
e^{\mathrm{T}} x=k \rho_{k}-(t-k)\left(m_{k}-1\right), \quad \frac{e^{\mathrm{T}} x}{t-k}+a=\rho_{k}\left(\frac{k}{t-k}+2\right) .
$$

Taking the inner product of relation (c) with the all-ones vector and using (42)(a) and (43), we find:

$$
\begin{aligned}
0 & =e^{\mathrm{T}} A x+a e^{\mathrm{T}} y=e^{\mathrm{T}} A\left(\rho_{k} e-\frac{1}{t-k} C_{k} e\right)+a e^{\mathrm{T}} y \\
& =\rho_{k} e^{\mathrm{T}} A e-\frac{1}{t-k} e^{\mathrm{T}}\left(I-y x^{\mathrm{T}}\right) e+a e^{\mathrm{T}} y=\rho_{k} e^{\mathrm{T}} A e-\frac{k}{t-k}+e^{\mathrm{T}} y\left(\frac{x^{\mathrm{T}} e}{t-k}+a\right) \\
& =\rho_{k}\left(e^{\mathrm{T}} A e+2 e^{\mathrm{T}} y\right)+\frac{k}{t-k}\left(\rho_{k} e^{\mathrm{T}} y-1\right) ;
\end{aligned}
$$

that is,

$$
e^{\mathrm{T}} A e+2 e^{\mathrm{T}} y=\frac{k}{t-k}\left(\frac{1}{\rho_{k}}-e^{\mathrm{T}} y\right) .
$$

Using relations (42)(d),(b) and (43), we find

$$
\begin{aligned}
1= & x^{\mathrm{T}} y+a b=\left(\rho_{k} e-\frac{1}{t-k} C_{k} e\right)^{\mathrm{T}} y+a b \\
& =\rho_{k} e^{\mathrm{T}} y+\frac{b}{t-k} e^{\mathrm{T}} x+a b=\rho_{k} e^{\mathrm{T}} y+b \rho_{k}\left(\frac{k}{t-k}+2\right) ;
\end{aligned}
$$

that is,

$$
e^{\mathrm{T}} y=\frac{1}{\rho_{k}}-b\left(\frac{k}{t-k}+2\right)
$$

Relations (44) and (45) imply that $e^{\mathrm{T}}\left(C_{k+1}\right)^{-1} e=e^{\mathrm{T}} A e+2 e^{\mathrm{T}} y+b=b \frac{t^{2}}{(t-k)^{2}}$. By the cofactor rule, $b=\frac{\operatorname{det} C_{k}}{\operatorname{det} C_{k+1}}$, and the lemma follows.

Corollary 4 Let $k \geq 2$ and assume that $C_{k}(t)$ is nonsingular. Then,

$$
\operatorname{det} C_{k+1}(t)=\frac{2 t \rho_{k}}{t-k} \operatorname{det} C_{k}(t)-\frac{t^{2} \rho_{k}^{2}}{(t-k+1)^{2}} \operatorname{det} C_{k-1}(t),
$$

after setting $\rho_{k}:=m_{k} \frac{t-k-1}{2}$. 
Proof Setting $P:=\left(\begin{array}{cc}I & -\frac{1}{t-k} e \\ 0 & 1\end{array}\right)$, we find that $P^{\mathrm{T}} C_{k+1} P=\left(\begin{array}{cc}C_{k} & \rho_{k} e \\ \rho_{k} e^{\mathrm{T}} & \mu\end{array}\right)$, after setting $\mu:=m_{k} \frac{t(t-k-1)}{t-k}$. Set $u:=\left(C_{k}\right)^{-1} e$ and let $v_{1}, \ldots, v_{k+1}$ denote the columns of $P^{\mathrm{T}} C_{k+1} P$. Then, $v_{k+1}-\rho_{k}\left(\sum_{i=1}^{k} u_{i} v_{i}\right)$ has all zero entries except the last $(k+1)$-th entry equal to $\mu-\rho_{k}^{2}\left(\sum_{i=1}^{k} u_{i}\right)=m_{k} \frac{t(t-k-1)}{t-k}-\rho_{k}^{2} e^{\mathrm{T}}\left(C_{k}\right)^{-1} e$. Therefore, we can conclude that

$$
\operatorname{det} C_{k+1}=\operatorname{det} P^{\mathrm{T}} C_{k+1} P=\left(\frac{2 t \rho_{k}}{t-k}-\rho_{k}^{2} e^{\mathrm{T}}\left(C_{k}\right)^{-1} e\right) \operatorname{det} C_{k} .
$$

Relation (46) now follows directly from Lemma 8 and (47).

Lemma 9 Consider the rational functions $f_{1}(t)=t-1, f_{2}(t):=\frac{t^{2}(t-2)(3 t-2)}{4(t-1)^{2}}$ and, for $h=2, \ldots, k$,

$$
f_{h+1}(t)=\frac{2 t \rho_{h}}{t-h} f_{h}(t)-\frac{t^{2} \rho_{h}^{2}}{(t-h+1)^{2}} f_{h-1}(t),
$$

and the polynomials $g_{1}(t):=1, g_{2}(t):=3 t-2$ and, for $h=2, \ldots, k$,

$$
g_{h+1}(t)=\epsilon_{h}(t-h) g_{h}(t)-t(t-h-1) g_{h-1}(t),
$$

with $\epsilon_{h}=1$ if $h$ is even and $\epsilon_{h}=4$ otherwise. As before, $\rho_{h}:=m_{h} \frac{t-h-1}{2}$.

(i) For $h=2, \ldots, k+1, f_{h}(t)=\frac{\left.t^{h+1}\right)-1(t-h)}{4^{[h / 2]}(t-1)^{h}(t-2)^{h-1} \ldots(t-h+1)^{2}} g_{h}(t)$.

(ii) For $1 \leq k \leq 6, g_{k}(t)>0$ for all $t \geq k$. Moreover, $g_{7}(8)>0$.

Proof The proof for (i) is by induction on $k$. For (ii), setting $G_{k}(t):=g_{k}(t+k)$, one has to show that $G_{k}(t)>0$ for $t \geq 0, k \leq 6$. This follows from the fact that $G_{2}(t)=4+3 t, G_{3}(t)=7+7 t+2 t^{2}, G_{4}(t)=64+68 t+30 t^{2}+5 t^{3}, G_{5}(t)=$ $167+165 t+84 t^{2}+25 t^{3}+3 t^{4}, G_{6}(t)=1,776+1,296 t+540 t^{2}+248 t^{3}+70 t^{4}+7 t^{5}$. Moreover, $g_{7}(8)=1,024$.

We can now conclude the proof of Theorem 2. Let $t \geq r+1$. Consider first the case when $1 \leq r \leq \min (\alpha(G)-1,6)$. We show that (40) holds using Corollary 4 and Lemma 9. First note that $\operatorname{det} C_{h}(t)=f_{h}(t)$ for $h=1,2$ (direct verification). Let $k \in\{1, \ldots, r\}$. If $k=1,2$, then $\operatorname{det} C_{k}(t)>0$. Assume $k \geq 3$ and $C_{k-1}(t) \succ 0$. By Corollary $4, \operatorname{det} C_{1}(t), \ldots, \operatorname{det} C_{k}(t)$ are related via (46); that is, det $C_{h}(t)=f_{h}(t)$ for $h=1, \ldots, k$. We now deduce from Lemma 9 that $\operatorname{det} C_{k}(t)>0$. This shows that $C_{r}(t) \succ 0$ for $t \geq r+1$, which concludes the proof of the first part of Theorem 2 .

Consider now the case when $r=7=\alpha(G)-1$. We have to show that the matrix $M=t\left(I+A_{G}\right)-J$ from (25) with $t:=\alpha(G)=8$ belongs to $\mathcal{K}_{n}^{(7)}$. As before we are left with the task of proving that $\operatorname{det} C_{1}(t), \ldots, \operatorname{det} C_{7}(t)>0$ for $t=8$, which follows from the assertions $g_{1}(8), \ldots, g_{6}(8), g_{7}(8)>0$ in Lemma 9. This concludes the proof of Theorem 2 . 
Note that the same argument cannot be used for proving Conjecture 1 in the case $\alpha(G)=9$, since $g_{1}(9), \ldots, g_{6}(9)>0$ while $g_{7}(9)<0$ which implies that the matrix $C_{7}(9)$ is not positive semidefinite.

\section{Proof of Theorem 3}

The proof is along the same lines as in the preceding section. Set

$$
t:=\max _{i \in V} w_{i}+\vartheta_{w}^{(0)}\left(G \backslash i^{\perp}\right) .
$$

For $i \in V$, define $w^{(i)}:=\left(w_{j}\right)_{j \in V \backslash i^{\perp}}$, the vector of node weights restricted to the graph $G \backslash i^{\perp}$. The matrix $P_{i}:=\left(t-w_{i}\right)\left(\operatorname{Diag}\left(w^{(i)}\right)+W_{G} A_{G \backslash i^{\perp}}\right)-w^{(i)}\left(w^{(i)}\right)^{\mathrm{T}}$ belongs to the cone $K_{\left|V \backslash i^{\perp}\right|}^{(0)}\left(\right.$ since $\left.W_{G \backslash i^{\perp}} \leq W_{G}\right)$. Define $a:=\left(1 / w_{j}\right)_{j \in V}$ and, for $i \in V$, let $a^{(i)}$ be the restriction of $a$ to $G \backslash i^{\perp}$. Moreover, set $B_{G \backslash i^{\perp}}:=$ $W_{G} \operatorname{Diag}\left(a^{(i)}\right) A_{G \backslash i^{\perp}} \operatorname{Diag}\left(a^{(i)}\right)$ and $M_{i}:=\operatorname{Diag}\left(a^{(i)}\right) P_{i} \operatorname{Diag}\left(a^{(i)}\right)$. Then,

$$
M_{i}=\left(t-w_{i}\right)\left(\operatorname{Diag}\left(a^{(i)}\right)+B_{G \backslash i^{\perp}}\right)-J \in K_{\left|V \backslash i^{\perp}\right|}^{(0)} .
$$

Our goal is to show that $P:=t\left(\operatorname{Diag}(w)+W_{G} A_{G}\right)-w w^{\mathrm{T}} \in \mathcal{K}_{n}^{(1)}$; equivalently, setting $M:=\operatorname{Diag}(a) P \operatorname{Diag}(a)$, we have to show that

$$
\text { the polynomial } p(x):=\left(\sum_{i} a_{i} x_{i}^{2}\right) v(x)^{\mathrm{T}} M v(x) \text { is a SOS. }
$$

We follow the same strategy as in the proof of Theorem 2: we introduce a set of symmetric matrices $X(i)(i \in V)$ satisfying (26). Namely, given $i \in V$, all entries of $X(i)$ are equal to 0 , except

$$
\begin{aligned}
& X(i)_{i j}=-\frac{1}{2} a_{j}\left(a_{i}-a_{j}\right) t, \quad X(i)_{j j}=a_{i}\left(a_{j}-a_{i}\right) t \text { for } j \in N(i), \\
& X(i)_{j k}=-a_{i} a_{j} a_{k} W_{G} t \text { for } j, k \in N(i), j \not z k, \\
& X(i)_{j k}=a_{i} a_{j} a_{k} W_{G} t \text { for } j \in N(i), k \in V \backslash i^{\perp}, j \simeq k .
\end{aligned}
$$

Then, $X(i)_{j k}+X(j)_{i k}+X(k)_{i j} \geq 0$ since it takes the values

- For $i=j=k: 0$;

- For $i=j \simeq k: 2\left(-\frac{1}{2} a_{k}\left(a_{i}-a_{k}\right) t\right)+a_{k}\left(a_{i}-a_{k}\right) t=0$;

- For $i=j \not z k: 2 \cdot 0+0=0$;

- For $i \simeq j \simeq k \simeq i: 0+0+0=0$;

- For $i \simeq j \not z k \simeq i:-a_{i} a_{j} a_{k} W_{G} t+a_{i} a_{j} a_{k} W_{G} t+a_{i} a_{j} a_{k} W_{G} t=a_{i} a_{j} a_{k} W_{G} t$;

- For $i \not z j \simeq k \not ॅ i: 0+0+0=0$;

- For $i \not z j \not z k \not z i: 0+0+0=0$. 
One can decompose the polynomial $p(x)$ as

$$
\sum_{i} x_{i}^{2} v(x)^{\mathrm{T}}\left(a_{i} M-X(i)\right) v(x)+\sum_{i} x_{i}^{2} v(x)^{\mathrm{T}} X(i) v(x)
$$

The second summation is a SOS by Lemma 5. To conclude the proof, it suffices to show that the matrix $a_{i} M-X(i)$ belongs to $\mathcal{K}_{n}^{(0)}$. For this, note that the matrix $A:=w_{i}\left(a_{i} M-X(i)\right)=M-w_{i} X(i)$ can be decomposed as

$$
A=\left(\begin{array}{cc}
\left(t a_{i}-1\right) J & -J \\
-J & \frac{1}{t a_{i}-1} J
\end{array}\right)+\left(\begin{array}{cc}
N & 0 \\
0 & 0
\end{array}\right)+\left(\begin{array}{cc}
0 & 0 \\
0 & \frac{t a_{i}}{t a_{i}-1} M_{i}
\end{array}\right)
$$

where $N \geq 0$, which shows that $A \in \mathcal{K}_{n}^{(0)}$ and concludes the proof of Theorem 3. (When all weights are equal to $1, N=0$ and we find the decomposition from (29), (30).) Indeed, one can verify that

$$
\begin{aligned}
& A_{j j}=t a_{i}-1 \text { for } j \in i^{\perp} \\
& A_{i j}=t W_{G} a_{i} a_{j}-1+\frac{a_{j}}{2 a_{i}}\left(a_{i}-a_{j}\right) t \text { for } j \in N(i) \\
& A_{j k}=t W_{G} a_{j} a_{k}-1 \quad \text { for } j \neq k \in N(i) \\
& A_{j k}=-1 \text { for } j \in i^{\perp}, k \in V \backslash i^{\perp} \\
& A_{j j}=t a_{j}-1 \text { for } j \in V \backslash i^{\perp} \\
& A_{j k}=t W_{G} a_{j} a_{k}-1 \text { for } j \neq k \in V \backslash i^{\perp}, j \simeq k \\
& A_{j k}=-1 \quad \text { for } j, k \in V \backslash i^{\perp}, j \not k
\end{aligned}
$$

The principal submatrix of $A$ indexed by $V \backslash i^{\perp}$ is thus equal to $\frac{1}{t a_{i}-1} J+\frac{t a_{i}}{t a_{i}-1} M_{i}$. Moreover, $t W_{G} a_{j} a_{k}-1 \geq t a_{i}-1$ since $W_{G} \geq \frac{a_{i}}{a_{j} a_{k}}=\frac{w_{j} w_{k}}{w_{i}}$. Finally, we have $t W_{G} a_{i} a_{j}-1+\frac{a_{j}}{2 a_{i}}\left(a_{i}-a_{j}\right) t \geq t a_{i}-1$ since $W_{G} \geq \frac{a_{j}^{2}+2 a_{i}^{2}-a_{i} a_{j}}{2 a_{i}^{2} a_{j}}=\frac{w_{i}^{2}+2 w_{j}^{2}-w_{i} w_{j}}{2 w_{j}} ;$ indeed, if $w_{i} \leq w_{j}$, then $\frac{w_{i}^{2}+2 w_{j}^{2}-w_{i} w_{j}}{2 w_{j}} \leq w_{j} \leq w_{\max } \leq W_{G}$ and, if $w_{i} \geq w_{j}$, then $\frac{w_{i}^{2}+2 w_{j}^{2}-w_{i} w_{j}}{2 w_{j}} \leq \frac{w_{i}\left(w_{i}+w_{j}\right)}{2 w_{j}} \leq \frac{\left(w_{\max }\right)^{2}}{w_{\min }}=W_{G}$.

\section{Proof of Theorem 4}

Obviously, las ${ }^{(1)}=\vartheta^{(0)}(G)$. In view of Lemma 2, we have to show that las ${ }^{(r)} \leq$ $\tilde{\vartheta}^{(r-1)}(G)$ for any positive integer $r$. For this, let $x \in \mathbb{R}^{\mathcal{P}_{2 r}(V)}$ be feasible for (2), i.e., $x_{\emptyset}=1, x_{I} \geq 0(|I|=r+1), x_{i j}=0(i j \in E)$, and $M_{r}(x) \succeq 0$. Then, $x_{I}=0$ for any $I \in \mathcal{P}_{2 r}(V)$ containing an edge. We may assume that $\sum_{i=1}^{n} x_{i}>0$. For $p=1, \ldots, r+1$, define

$$
\ell_{p}:=\sum_{\beta \in I(n, p-1)} \frac{(p-1) !}{\beta !} x_{S(\beta)}
$$


Then, $\ell_{1}=1, \ell_{p} \geq \ell_{2}=\sum_{i=1}^{n} x_{i}>0$ for $p \geq 2$. For $p=1, \ldots, r$, define $y=\left(y_{\delta}\right)_{\delta \in I(n, 2 p+2)}$ as follows: $y_{\delta}=0$ if $S_{\text {odd }}(\delta) \neq \emptyset, y_{\delta}:=\frac{1}{\ell_{p}} x_{S(\delta)}$ otherwise (then $|S(\delta)| \leq p+1 \leq r+1$ ).

Lemma $10 N_{p+1}(y) \succeq 0$.

Proof For $I \subseteq V$, set $\mathcal{O}_{I}:=\left\{\beta \in I(n, p+1) \mid S_{\text {odd }}(\beta)=I\right\}$ and $N_{I}:=$ $\left(y_{\beta+\beta^{\prime}}\right)_{\beta, \beta^{\prime} \in \mathcal{O}_{I}}$. Then, $N_{p+1}(y)$ is a block diagonal matrix with the matrices $N_{I}$ $(I \subseteq V)$ as diagonal blocks. As $\ell_{p} N_{I}=\left(x_{S(\beta) \cup S\left(\beta^{\prime}\right)}\right)_{\beta, \beta^{\prime} \in \mathcal{O}_{I}}, N_{I} \succeq 0$ since it is obtained from a principal submatrix of $M_{r}(x)$ by duplicating certain rows/columns (unless $|I|=r+1$ in which case $N_{I}$ is the $1 \times 1$ matrix with entry $x_{|I|} \geq 0$, implying again $N_{I} \succeq 0$ ).

Therefore, the matrix $Z(p):=C(y)=\sum_{\gamma \in I(n, p-1)} \frac{(p-1) !}{\gamma !} N^{\gamma}(y)$ belongs to the cone $\mathcal{C}_{n}^{(p-1)}$. Moreover, $Z(p)_{i j}=0$ if $i j \in E$. Define the matrix

$$
\tilde{Z}(p):=\left(\begin{array}{cc}
1 & Z(p)_{11} \ldots Z(p)_{n n} \\
Z(p)_{11} & \\
\vdots & Z(p) \\
Z(p)_{n n} &
\end{array}\right) .
$$

Lemma $11 \tilde{Z}(p) \succeq 0$.

Proof The matrix

$$
\begin{array}{r}
\ell_{p} \tilde{Z}(p)=\sum_{\gamma \in I(n, p-1)} \frac{(p-1) !}{\gamma !}\left(\begin{array}{cc}
x_{S(\gamma)} & y_{2 \gamma+4 e_{1}} \ldots y_{2 \gamma+4 e_{n}} \\
y_{2 \gamma+4 e_{1}} & \\
\vdots & \left(y_{2 \gamma+2 e_{j}+2 e_{k}}\right)_{j, k=1}^{n} \\
y_{2 \gamma+4 e_{n}} &
\end{array}\right) \\
=\sum_{\gamma \in I(n, p-1)} \frac{(p-1) !}{\gamma !}\left(\begin{array}{cc}
x_{S(\gamma)} & x_{S\left(\gamma+e_{1}\right)} \ldots x_{S\left(\gamma+e_{n}\right)} \\
x_{S\left(\gamma+e_{1}\right)} & \\
\vdots & \left(x_{S\left(\gamma+e_{j}+e_{k}\right)}\right)_{j, k=1}^{n} \\
x_{S\left(\gamma+e_{n}\right)} &
\end{array}\right)
\end{array}
$$

is positive semidefinite, since the matrices in the above summation are principal submatrices of $M_{r}(x)$.

Lemma $12 \operatorname{Tr}(J Z(p))=\frac{\ell_{p+2}}{\ell_{p}}$ and $\operatorname{Tr}(Z(p))=\frac{\ell_{p+1}}{\ell_{p}}$.

Proof As $Z(p)=C(y) \in \mathcal{C}_{n}^{(p-1)}$, one can use (14) and (15). Namely,

$$
\operatorname{Tr}(J Z(p))=\sum_{\beta \in I(n, p+1)} \frac{(p+1) !}{\beta !} y_{2 \beta}=\frac{1}{\ell_{p}} \sum_{\beta \in I(n, p+1)} \frac{(p+1) !}{\beta !} x_{S(\beta)}=\frac{\ell_{p+2}}{\ell_{p}} .
$$


Moreover,

$$
\begin{gathered}
\operatorname{Tr}(Z(p))=\sum_{\beta \in I(n, p+1)} \frac{(p-1) !}{\beta !} y_{2 \beta} \sum_{i=1}^{n}\left(\beta_{i}^{2}-\beta_{i}\right) \\
=\frac{1}{\ell_{p}} \sum_{i=1}^{n} \sum_{\beta \in I(n, p+1)} \frac{(p-1) !}{\beta !} \beta_{i}\left(\beta_{i}-1\right) x_{S(\beta)} .
\end{gathered}
$$

We can restrict the inner summation to $\beta$ with $\beta_{i} \geq 2$. Then, $\delta:=\beta-e_{i}$ has the same support as $\beta$ and

$$
\operatorname{Tr}(Z(p))=\frac{1}{\ell_{p}} \sum_{i=1}^{n} \sum_{\delta \in I(n, p)} \frac{(p-1) !}{\delta !} \delta_{i} x_{S(\delta)}=\frac{1}{\ell_{p}} \sum_{\delta \in I(n, p)} \frac{(p-1) !}{\delta !}|\delta| x_{S(\delta)}=\frac{\ell_{p+1}}{\ell_{p}} .
$$

Lemma $13 \frac{\ell_{p+2}}{\ell_{p+1}} \geq \frac{\ell_{p+1}}{\ell_{p}}$.

Proof By Lemma 11, $\tilde{Z}(p) \succeq 0$, implying $Z(p)-\operatorname{diag}(Z(p)) \operatorname{diag}(Z(p))^{\mathrm{T}} \succeq 0$. Therefore, $e^{\mathrm{T}}\left(Z(p)-\operatorname{diag}(\bar{Z}(p)) \operatorname{diag}(Z(p))^{\mathrm{T}}\right) e \geq 0$, yielding $\operatorname{Tr}(J Z(p)) \geq$ $(\operatorname{Tr}(Z(p)))^{2}$. The result now follows using Lemma 12 .

From Lemmas 12 and 13, we deduce that $\sum_{i=1}^{n} Z(r)_{i i}=\frac{\ell_{r+1}}{\ell_{r}} \geq \frac{\ell_{2}}{\ell_{1}}=\sum_{i=1}^{n} x_{i}$. The vector $z:=\operatorname{diag}(Z(r))$ is feasible for the program (16) defining the parameter $\tilde{\vartheta}^{(r-1)}(G)$. Hence, $\tilde{\vartheta}^{(r-1)}(G) \geq \sum_{i=1}^{n} z_{i}=\operatorname{Tr}(Z(r)) \geq \sum_{i=1}^{n} x_{i}$. This shows that $\tilde{\vartheta}^{(r-1)}(G) \geq \operatorname{las}^{(r)}(G)$.

\section{Concluding remarks}

6.1 Some variations of the bound $\vartheta^{(r)}(G)$

Given a polynomial $q \in \mathbb{R}\left[x_{1}, \ldots, x_{n}\right]$, define the even polynomial $\tilde{q}(x):=$ $q\left(x_{1}^{2}, \ldots, x_{n}^{2}\right)$; a polynomial being even when each variable occurs with an even degree in any nonzero term. One can express the condition that $\tilde{q}$ be a SOS directly in terms of the polynomial $q$.

Proposition 2 [26] Given a homogeneous polynomial $q$ of degree $d$, the associated even polynomial $\tilde{q}(x):=q\left(x_{1}^{2}, \ldots, x_{n}^{2}\right)$ is a SOS if and only if the polynomial $q$ admits a decomposition:

$$
q(x)=\sum_{\substack{I \subseteq\{1, \ldots, n\} \\|I| \leq d,|I| \equiv d(\bmod 2)}} \sigma_{I}(x) \prod_{i \in I} x_{i}
$$

where $\sigma_{I}$ is a form of degree $d-|I|$ which is SOS. 
The condition (49) can obviously be reformulated as

$$
q(x)=\sum_{\beta \in \mathbb{Z}_{+}^{n},|\beta| \leq d,|\beta| \equiv d \bmod 2} \sigma_{\beta} x^{\beta}
$$

where $\sigma_{\beta}$ is a form of degree $d-|\beta|$ which is SOS. As the polynomial $p_{M}^{(r)}$ from (4) is an even polynomial, we can apply the above criterion for characterizing whether an $n \times n$ matrix $M$ belongs to the cone $\mathcal{K}_{n}^{(r)}$. Namely, $M \in \mathcal{K}_{n}^{(r)}$ if and only if

$$
\left(\sum_{i=1}^{n} x_{i}\right)^{r} x^{\mathrm{T}} M x=\sum_{\beta \in \mathbb{Z}_{+}^{n},|\beta| \leq r+2,|\beta| \equiv r+2 \bmod 2} \sigma_{\beta} x^{\beta}
$$

where $\sigma_{\beta}$ is a form of degree $r+2-|\beta|$ which is SOS.

Peña et al. [17] consider the set $\mathcal{Q}_{n}^{(r)}$ consisting of the matrices $M$ for which such a decomposition (51) exists involving only the two highest order terms with $|\beta|=r+2, r$. Therefore, $\mathcal{Q}_{n}^{(r)}$ is a subcone of the cone $\mathcal{K}_{n}^{(r)}$ with equality $\mathcal{Q}_{n}^{(r)}=\mathcal{K}_{n}^{(r)}$ for $r=0,1$, and the bound

$$
v^{(r)}(G):=\min t \quad \text { such that } t\left(I+A_{G}\right)-J \in \mathcal{Q}_{n}^{(r)}
$$

satisfies

$$
\alpha(G) \leq \vartheta^{(r)}(G) \leq v^{(r)}(G)
$$

For $r \leq \alpha(G)-1$, Peña et al. [17] show that

$$
v^{(r)}(G) \leq r+\max _{S \subseteq V, S \text { stable, }|S|=r} v^{(0)}\left(G \backslash S^{\perp}\right)
$$

for $r=1,2,3$, and for $r=4,5$ if $\alpha(G) \leq 6$, which implies $v^{(r)}(G)=\alpha(G)$ if $\alpha(G) \leq 6$, thus proving Conjecture 1 for graphs with $\alpha(G) \leq 6$.

For $r \leq \alpha(G)-1$, our proof of Theorem 2 shows in fact that relation (53) holds for $r \leq 6$, and for $r=7$ if $\alpha(G)=8$. Indeed, the decomposition (31) shows that the matrix $M$ from (25) belongs to the cone $\mathcal{Q}_{n}^{(r)}$. This implies $v^{(\alpha(G)-1)}(G)=\alpha(G)$ if $\alpha(G) \leq 8$.

It is known (see [5]) that, for the circuit $C_{5}$ on 5 nodes, $\alpha\left(C_{5}\right)=2=$ $\vartheta^{(1)}\left(C_{5}\right)<\vartheta^{(0)}\left(C_{5}\right)$. Peña et al. [17] construct graphs $G_{8}, G_{11}, G_{14}$ with, respectively, 5,8,11,14 nodes, that satisfy: $\alpha\left(G_{n}\right)=\vartheta^{(2)}\left(G_{n}\right)$ for $n=8,11,14$, $\alpha\left(G_{8}\right)=3<v^{(1)}\left(G_{8}\right), \alpha\left(G_{11}\right)=4<v^{(2)}\left(G_{11}\right)$, and $\alpha\left(G_{14}\right)=5<v^{(3)}\left(G_{14}\right)$. Therefore, the inclusion $\mathcal{Q}_{n}^{(r)} \subseteq \mathcal{K}_{n}^{(r)}$ is strict for $r=2$.

Let us mention a consequence of the strict inclusion $\mathcal{Q}_{n}^{(2)} \subset \mathcal{K}_{n}^{(2)}$ for the description of the cone $\mathcal{K}_{n}^{(2)}$. The following sufficient condition for membership in $\mathcal{K}_{n}^{(r)}$ has already been implicitly mentioned earlier in the paper (e.g., in Sect. 3.2). 
Lemma 14 Let $M$ be a symmetric $n \times n$ matrix and $r \geq 1$ an integer. If there exist matrices $X(1), \ldots, X(n)$ satisfying (26) and for which $M-X(i) \in \mathcal{K}_{n}^{(r-1)}$ (for $i=1, \ldots, n)$, then $M \in \mathcal{K}_{n}^{(r)}$.

Proof Directly from the decomposition (28).

For $r=1, \mathcal{K}_{n}^{(1)}=\mathcal{Q}_{n}^{(1)}$ and the implication of Lemma 14 holds as an equivalence, which gives the characterization of the cone $\mathcal{K}_{n}^{(1)}$ from $[1,16]$. For $r=2$ however, the reverse implication does not hold since $\mathcal{Q}_{n}^{(2)}$ is a strict subset of $\mathcal{K}_{n}^{(2)}$.

In order to prove relation (21) for any $r$ and thus Conjecture 1, one should probably obtain a decomposition (51) for the polynomial $\left(\sum_{i} x_{i}\right)^{r} x^{\mathrm{T}} M x$ involving also terms with $|\beta|<r$ (while our proof in this paper involves only terms with $|\beta|=r, r+2)$.

Note finally that one can formulate the following sharpening of the bound $\vartheta^{(r)}(G)$

$$
\underline{\vartheta}^{(r)}(G):=\min _{s, t \in \mathbb{R}} t \quad \text { subject to } t I+s A_{G}-J \in \mathcal{K}_{n}^{(r)}
$$

whose dual formulation reads

$$
\max \operatorname{Tr}(J X) \quad \text { subject to } \operatorname{Tr}(X)=1, \operatorname{Tr}\left(A_{G} X\right)=0, X \in \mathcal{C}_{n}^{(r)}=\left(\mathcal{K}_{n}^{(r)}\right)^{*}
$$

and is obtained by splitting the constraint $\operatorname{Tr}\left(\left(I+A_{G}\right) X\right)=1$ into $\operatorname{Tr}(X)=1$ and $\operatorname{Tr}\left(A_{G} X\right)=0$. The bounds $\tilde{\vartheta}^{(r)}(G)$ (from $(16)$ ), $\underline{\vartheta}^{(r)}(G)$ (from (54)) and $\vartheta^{(r)}(G)$ (from (6), (10)) satisfy

$$
\tilde{\vartheta}^{(r)}(G) \leq \underline{\vartheta}^{(r)}(G) \leq \vartheta^{(r)}(G)
$$

The second inequality is obvious. For the first one take a matrix $X$ that corresponds to $x$ feasible for (16). Then $\frac{X}{\operatorname{Tr}(X)}$ is feasible for (55) and $\frac{\operatorname{Tr}(J X)}{\operatorname{Tr}(X)}=$ $\frac{\operatorname{Tr}(J X)}{\sum_{i=1}^{n} x_{i}} \geq \sum_{i=1}^{n} x_{i}$ since $X-x x^{\mathrm{T}} \succeq 0$.

For $r=0$, the three bounds coincide ([5], see also Lemma 4). It is not clear whether they coincide for $r \geq 1$.

\subsection{Some variations of Conjecture 1}

The next lemma shows that a block matrix belongs to the cone $\mathcal{K}^{(r)}$ if and only if its skeleton does. Combined with arguments in our proof of Theorem 2, it will enable us to derive Proposition 3 below.

Lemma 15 Consider the matrices

$$
M=\left(\begin{array}{cc}
A & b \\
b^{\mathrm{T}} & c
\end{array}\right), \quad M^{\prime}=\left(\begin{array}{lll}
A & b & b \\
b^{\mathrm{T}} & c & c \\
b^{\mathrm{T}} & c & c
\end{array}\right)
$$

with respective sizes $n$ and $n+1$. Then, $M \in \mathcal{K}_{n}^{(r)} \Longleftrightarrow M^{\prime} \in \mathcal{K}_{n+1}^{(r)}$. 
Proof Assume that $M \in \mathcal{K}_{n}^{(r)}$. Then, the polynomial $\left(\sum_{i=1}^{n} y_{i}\right)^{r} y^{\mathrm{T}} M y$ (in the variables $y_{1}, \ldots, y_{n}$ ) has a decomposition of the form (51). In view of the shape of the matrix $M^{\prime}$, the polynomial $\left(\sum_{i=1}^{n+1} x_{i}\right)^{r} x^{\mathrm{T}} M^{\prime} x$ (in the variables $x_{1}, \ldots, x_{n}$, $\left.x_{n+1}\right)$ can be written as $\left(\sum_{i=1}^{n} y_{i}\right)^{r} y^{\mathrm{T}} M y$, after setting $y_{i}=x_{i}$ for $i \leq n-1$ and $y_{n}=x_{n}+x_{n+1}$. Therefore, $\left(\sum_{i=1}^{n+1} x_{i}\right)^{r} x^{\mathrm{T}} M^{\prime} x$ also admits a decomposition of the form (51), which shows that $M^{\prime} \in \mathcal{K}_{n+1}^{(r)}$. The reverse implication is easy (simply set the additional variable to zero).

Proposition 3 For a graph $G=(V, E)$ and an integer $r \geq 1$, we have

$$
\vartheta^{(r)}(G) \leq \max _{i \in V} \vartheta^{(r-1)}(G \backslash N(i)) .
$$

Proof Set $t:=\max _{i \in V} \vartheta^{(r-1)}(G \backslash N(i))$. Then, for any $i \in V$, the matrix

$$
t\left(I+A_{G \backslash N(i)}\right)-J={ }_{V \backslash i^{\perp}}^{i}\left(\begin{array}{cc}
i & V \backslash i^{\perp} \\
t-1 & -e^{\mathrm{T}} \\
-e & t\left(I+A_{G \backslash i^{\perp}}\right)-J
\end{array}\right)
$$

belongs to the cone $\mathcal{K}_{|V \backslash N(i)|}^{(r-1)}$. Lemma 15 implies that the matrix

$$
M-X(i)=i^{\perp} \quad V \backslash i^{\perp}\left(\begin{array}{cc}
i^{\perp} & V \backslash i^{\perp} \\
(t-1) J & -J \\
-J & t\left(I+A_{G \backslash i^{\perp}}\right)-J
\end{array}\right)
$$

from (29) belongs to $\mathcal{K}_{n}^{(r-1)}$. In view of (28), this implies that $M \in \mathcal{K}_{n}^{(r)}$.

We can now formulate some strengthenings of Conjecture 1.

Conjecture 2 For any $r \geq 1$,

$$
\vartheta^{(r)}(G) \leq r+\max _{S \subseteq V, S \text { stable, }|S|=r} \vartheta^{(0)}\left(G \backslash S^{\perp}\right) .
$$

Conjecture 3 For any $r \geq 1$,

$$
\vartheta^{(r)}(G) \leq 1+\max _{i \in V} \vartheta^{(r-1)}\left(G \backslash i^{\perp}\right) .
$$

Conjecture 4 If $i$ is an isolated node in $G$, i.e., $N(i)=\emptyset$, then for any $r \geq 0$,

$$
\vartheta^{(r)}(G) \leq \vartheta^{(r)}(G \backslash i)+1 .
$$

Theorem 2 claims that Conjecture 2 holds for $r \leq \min (6, \alpha(G)-1)$. For $r=1$ Conjectures 2 and 3 are identical; they hold by (9), which was first proved by 
de Klerk and Pasechnik [5]. Conjecture 4 is true for $r=0$. In order to prove this set $t:=1+\vartheta^{(0)}(G \backslash i)$. Then $(t-1)\left(I+A_{G \backslash i}\right)-J \in \mathcal{K}_{n-1}^{(0)}$. Observe that $M:=t\left(I+A_{G}\right)-J \in \mathcal{K}_{n}^{(0)}$ can be decomposed as in (29) (with $i^{\perp}=\{i\}$ as $i$ is isolated). The first matrix in this decomposition belongs to $\mathcal{K}_{n}^{(0)}$ since $\mathcal{K}_{n-1}^{(0)}$ is closed under adding a zero row/column. The second matrix is positive semidefinite.

Lemma 16 Conjecture $4 \Longrightarrow$ Conjecture $3 \Longrightarrow$ Conjecture $2 \Longrightarrow$ Conjecture 1 .

Proof The implication: Conjecture $4 \Longrightarrow$ Conjecture 3 follows using Proposition 3 , and the implications: Conjecture $3 \Longrightarrow$ Conjecture $2 \Longrightarrow$ Conjecture 1 are obvious.

Hence the whole question boils down to showing that the parameter $\vartheta^{(r)}(\cdot)$ behaves well on a graph with an isolated node. Note that the condition (59) is a natural requirement which holds, e.g., for the parameter $\alpha(\cdot)$ in place of $\vartheta^{(r)}(\cdot)$, or for the parameter las ${ }^{(r)}(\cdot)$ (easy to check). A reason why the parameter las ${ }^{(r)}(\cdot)$ is much easier to handle than the new bound $\vartheta^{(r)}(\cdot)$ might lie in the fact that the formulation of the Lasserre bound incorporates in an explicit way the $0 / 1$ condition, while the formulation of $\vartheta^{(r)}(\cdot)$ does not.

\subsection{Another interpretation of the bound $\vartheta^{(r)}(G)$}

We finally observe that the dual formulation $(10)$ for $\vartheta^{(r)}(G)$ can be interpreted as the Shor relaxation of a polynomial optimization program giving yet another formulation of $\alpha(G)$. For a graph $G=(V, E)$ and an integer $r \geq 0$, consider the program

$$
\max \left(\sum_{i \in V} x_{i}^{2}\right)^{r+2} \quad \text { subject to }\left(\sum_{i \in V} x_{i}^{2}\right)^{r} v(x)^{\mathrm{T}}\left(I+A_{G}\right) v(x)=1 .
$$

Lemma 17 For any integer $r \geq 0, \alpha(G)$ is equal to the optimum value of (60).

Proof Let $\mu$ denote the maximum value of (60). Given a stable set $S \subseteq V$, the vector $x:=t \chi^{S}$ where $t:=|S|^{-\frac{r+1}{2(r+2)}}$, is feasible for (60) with objective value $|S|$, which shows $\alpha(G) \leq \mu$. Conversely, if $x$ is feasible for (60), then $v(x)^{\mathrm{T}}\left(\alpha(G)\left(I+A_{G}\right)-J\right) v(x) \geq 0$, since the matrix $\alpha(G)\left(I+A_{G}\right)-J$ is copositive. This implies that $\mu \leq \alpha(G)$.

Following Lasserre [7], one can define a hierarchy of relaxations for the polynomial optimization problem (60). As the degree of the polynomials involved in (60) is $2 r+4$, the Shor relaxation of the problem (i.e., the relaxation of lowest order in the hierarchy; see [7] for details) reads

$$
\begin{array}{ll}
\max & \sum_{\beta \in I(n, r+2)} \frac{(r+2) !}{\beta !} y_{2 \beta} \\
\text { subject to } & N_{r+2}(y) \succeq 0 \\
& \sum_{\beta \in I(n, r+2)} \frac{r !}{\beta !}\left(\beta^{\mathrm{T}}\left(I+A_{G}\right) \beta-\beta^{\mathrm{T}} e\right) y_{2 \beta}=1 .
\end{array}
$$


(Recall the definition of the matrix $N_{r+2}(y)$ from Definition 1.) In view of (8) and (14), the objective function reads: $\operatorname{Tr}(J C(y))$ and the constraint reads: $\operatorname{Tr}\left(\left(I+A_{G}\right) C(y)\right)=1$. Therefore, the program (61) is identical to the program (10) giving the dual formulation of $\vartheta^{(r)}(G)$.

Acknowledgment This work was supported by the Netherlands Organization for Scientific Research grant NWO 639.032.203. The authors thank Etienne de Klerk for several valuable discussions about the topic of this paper and two referees for their careful reading and useful suggestions.

\section{References}

1. Bomze, I.M., Klerk, E. de : Solving standard quadratic optimization problems via linear, semidefinite and copositive programming. J. Global Optim. 24, 163-185 (2002)

2. Boyd, S., Vandenberghe, L.: Semidefinite programming. SIAM Rev. 38, 49-95 (1996)

3. Busygin, S.: A New Trust Region Technique for the Maximum Weight Clique Problem. Discrete Appl. Math. 154(15), 2080-2096 (2006)

4. Delsarte, P.: An Algebraic Approach to the Association Schemes of Coding Theory. [Philips Research Reports Supplements (1973) No. 10] Philips Research Laboratories, Eindhoven (1973)

5. De Klerk, E., Pasechnik, D.V.: Approximating the stability number of a graph via copositive programming. SIAM J. Optim. 12, 875-892 (2002)

6. Grötschel, M., Lovász, L., Schrijver, A.: Geometric Algorithms and Combinatorial Optimization. Springer, Berlin Heidelberg New York (1988)

7. Lasserre, J.B.: Global optimization with polynomials and the problem of moments. SIAM J. Optim. 11, 796-817 (2001)

8. Lasserre J.B. (2001) An explicit exact SDP relaxation for nonlinear 0 - 1 programs. In: Aardal K., Gerards A.M.H. (eds.) Lecture Notes in Computer Science, vol. 2081, pp. 293-303

9. Laurent, M.: A comparison of the Sherali-Adams, Lovász-Schrijver and Lasserre relaxations for $0-1$ programming. Math. Oper. Res. 28, 470-496 (2003)

10. Laurent, M.: Strengthened semidefinite programming bounds for codes. Math. Program. (in press) (2006)

11. Laurent, M., Rendl, F.: Semidefinite programming and integer programming. In: Aardal, K., Nemhauser, G., Weismantel, R. (eds.) Handbook on Discrete Optimization, pp 393-514 (2005)

12. Lovász, L.: On the Shannon capacity of a graph. IEEE Trans. Inf. Theory 25, 1-7 (1979)

13. Lovász, L., Schrijver, A.: Cones of matrices and set-functions and 0-1 optimization. SIAM J. Optim. 1, 166-190 (1991)

14. McEliece, R.J., Rodemich, E.R., Rumsey, H.C.: The Lovász' bound and some generalizations. J. Comb. Inf. Syst. Sci. 3, 134-152 (1978)

15. Motzkin, T.S., Straus, E.G.: Maxima for graphs and a new proof of a theorem of Túran. Can. J. Math. 17, 533-540 (1965)

16. Parrilo, P.A.: Structured semidefinite programs and semialgebraic geometry methods in robustness and optimization. $\mathrm{PhD}$ thesis, California Institute of Technology (2000)

17. Peña, J.F., Vera, J.C., Zuluaga, L.F.: Computing the stability number of a graph via linear and semidefinite programming. Preprint, 2005. Available at http://www.optimization-online. org/DB_HTML/2005/04/1106.html

18. Pólya, G.: Collected Papers, vol. 2, pp 309-313. MIT Press, Cambridge, Mass., London (1974)

19. Reznick, B.: Some concrete aspects of Hilbert's 17th problem. Preprint. Available at http://www.math.uiuc.edu/ reznick/

20. Reznick, B.: Sums of even powers of real linear forms. Memoirs of the American Mathematical Society, Number 463 (1992)

21. Schrijver, A.: A comparison of the Delsarte and Lovász bounds. IEEE Trans. Inf. Theory 25, 425-429 (1979)

22. Schrijver, A.: Combinatorial Optimization-Polyhedra and Efficiency. Springer, Berlin Heidelberg New York (2003) 
23. Schrijver, A.: New code upper bounds from the Terwiliger algebra and semidefinite programming. IEEE Trans. Inf. Theory 51, 2859-2866 (2005)

24. Schweighofer, M.: Optimization of polynomials on compact semialgebraic sets. SIAM J. Optim. 15(3), 805-825 (2005)

25. Wolkowicz, H., Saigal, R., Vandenberghe, L. (eds.) (2000) Handbook of Semidefinite Programming. Kluwer, Boston/Dordrecht/London

26. Zuluaga, L.F., Vera, J.C., Peña, J.F.: LMI approximations for cones of positive semidefinite forms. SIAM J. Optim. 16(4), 1076-1091 (2006) 